\title{
Zonificación de alternativas de conectividad ecológica, restauración y conservación en las microcuencas Curubital, Mugroso, Chisacá y Regadera, cuenca del río Tunjuelo (Distrito Capital de Bogotá), Colombia
}

Zonification of alternatives for ecological connectivity, restoration and conservation of the Curubital, Mugroso, Chisacá and Regadera microdrainages of the Tunjuelo River (Capital District of Bogotá), Colombia

\section{Paola Isaacs-Cubides, Ledy Trujillo y Vilma Jaimes}

\section{Resumen}

El Distrito Capital presenta una gran diversidad de coberturas naturales e intervenidas, que requieren acciones de conservación y restauración para la preservación de los bienes y servicios que presta y su biodiversidad. Sin embargo, es necesario contar con insumos espaciales que permitan ubicar las zonas que requieren de estas acciones y que a su vez sean fuente para la toma de decisiones en especial en términos de conservación y conectividad entre coberturas. Se desarrollaron tres modelos de resistencia o conductancia con base en la cuantificación de criterios físicos, bióticos y socioeconómicos, definidos mediante reunión con expertos e insumos cartográficos disponibles, que incluyeron además un análisis funcional relacionado con aspectos ecológicos de la vegetación y un grupo de mamíferos. Los modelos obtenidos muestran las zonas de mayor estado de conservación o menor resistencia para las especies y adicionalmente indica las zonas con mayor intervención y mayor resistencia, estas con el fin de realizar una zonificación de las actividades de restauración en especial en zonas de páramos y bosques transicionales altoandinos. Los modelos también complementan lo propuesto en la Estructura Ecológica Distrital correspondiente a la conectividad, representatividad y cantidad de áreas protegidas, ya que desde una mirada funcional y el análisis de la composición y configuración del paisaje, aporta otros insumos que complementan las estrategias planteadas.

Palabras clave. Conexión. Estructura ecológica distrital. Fragmentación. Modelos de resistencia. Zonificación.

\begin{abstract}
Bogotá has a wide variety of natural and impacted vegetation covers, which require conservation and restoration actions to preserve the goods and services they provide as well as their biodiversity. However, a spatial analysis is necessary to locate which areas require these actions that will also become a source of information for decision making, especially in terms of conservation and connectivity between vegetation covers. We develop three models of resistance or conductance based on the quantification of physical, biotic and socio-economic criteria, defined by meetings with experts, and available cartographic resources that includes a functional analysis based on the ecological aspects of the vegetation cover and a group of mammals. The models obtained show the best
\end{abstract}


conserved areas or least resistance for species and also indicate those areas with greater degradation and higher resistance. This information facilitates the zonification of restoration activities, particularly in mountain moors and transitional Andean forests. The models also complement the proposals made in the Ecological Structure of the District report with respect to connectivity, representativeness and number of protected areas, because from the functional view and the analysis of the composition and configuration of the landscape, it provides other inputs to complement the proposed strategies.

Key words. Connection. Ecological Structure of the district. Fragmentation. Resistance models. Zonation.

\section{Introducción}

En la actualidad las coberturas naturales se han visto eliminadas por diferentes actividades humanas como la urbanización, deforestación, minería, establecimiento de cultivos y ganadería, entre otras, las cuales causan efectos adversos para las coberturas remanentes y representan un gran riesgo para la sobrevivencia y mantenimiento de la biodiversidad. Esta pérdida ocasiona lo que se conoce como fragmentación del paisaje, lo cual representa una pérdida en las conexiones naturales que hay entre los ecosistemas, separando y afectando el hábitat de las especies y sus poblaciones y exponiéndolas a mayor riesgo de extinción (Forman 1995, Murcia 1995).

Una de las estrategias propuestas para contrarrestar la fragmentación, es la conectividad, definida como el grado en el cual el paisaje facilita o impide movimientos entre parches de hábitat (Taylor et al. 1993, 2006) y el cual determina que tan conectado es un paisaje (Urban y Keitt 2001), pretende preservar las conexiones existentes y restaurar las que han sido eliminadas para favorecer la sobrevivencia a largo plazo de las especies (Noss y Cooperrider 1994, Bennett 2006). Las ventajas de trabajar y abordar la conectividad son varias ya que al momento de incrementar la cobertura o mantenerla, se reducen los niveles de contaminación sobre las cuencas, disminuye las consecuencias de la presencia de efecto de borde; adicionalmente mejora la prestación de bienes y servicios ambientales y contribuye a mitigar eventos estocásticos como inundaciones o incendios (Meffe y Carroll 1997, Primack 2001, Beier et al. 2008). Adicionalmente, al garantizar la conectividad se brinda mayor oportunidad para conservar procesos biológicos naturales y facilita a las especies usar otros rangos geográficos en busca de un nicho climático más adecuado (Hargrove et al. 2004, Beier et al. 2008).

La conectividad del paisaje se puede evaluar espacialmente en términos de composición y configuración del paisaje, determinada por los elementos presentes en el paisaje y como están distribuidos en el espacio respectivamente (Bennett 2006). Igualmente, la conectividad se aborda en términos funcionales, de acuerdo a las necesidades de las especies como tal que van a usar la estructura física del paisaje; este componente es más complejo de abordar ya que está pensado en un organismo en particular y varía según las condiciones locales en cada región donde se vaya a trabajar (Bennett 2006, Taylor et al. 2006). La conectividad en el paisaje puede ser implementada por medio de diferentes elementos del paisaje dependiendo del objetivo de conservación, buscando que un organismo pueda dispersarse, colonizar o migrar (Noss y Cooperrider 1994, Sieving et al. 2000, Bennett 2006).

La conectividad puede ser abordada de diferentes formas, en muchos casos pensando en acciones de restauración - rehabilitación - recuperación (Isaacs y Ariza 2015) que mejoren las condiciones del paisaje, lo que puede incluir la reconversión de sistemas productivos o la disminución de las presiones que presentes el paisaje (Sguerra et al. 2011, Vargas y Ramírez 2014). Es importante considerar que la conectividad, puede actuar como filtro hacia un hábitat hostil (Doak 1995), puede facilitar la presencia 
de depredadores y especies exóticas, así como el intercambio de enfermedades (Medici et al. 2007) o con individuos de otras poblaciones por efecto de borde (Noss 1991, Murcia 1995, Theobald 2006).

Los modelos de conectividad más empleados desde el ámbito espacial se basan en una grilla de resistencia, donde expresan la dificultad, el costo energético o el riesgo de mortalidad asociado al movimiento de los organismos entre parches de hábitat que conforman un paisaje a través de esa grilla (Urban y Keitt 2001, Adriaensen et al. 2003, Hargrove et al. 2004, McRae et al. 2012). Esta técnica busca espacializar las zonas menos hostiles para el movimiento de las especies, construidas a partir de la unión de diferentes variables espaciales como por ejemplo cobertura, pendiente, altura, presencia de infraestructura humana, distancia a fuentes de agua, entre otros aspectos del hábitat que son importantes para las especies, con valores que se asignan a dichas variables según faciliten o impidan la dispersión de los individuos (Bennett 2006, Theobald 2006). Una vez se cuenta con esa grilla, es posible modelar diferentes formas de conectividad, ya sea para garantizar la conservación, promover la restauración, proponer mejores estrategias de uso, entre otras (Beier et al. 2011, McRae et al. 2012).

La conectividad y preservación de las áreas protegidas en Bogotá, se ha abordado por medio del diseño de la Estructura Ecológica Distrital (EED), definida como un modelo del encadenamiento espacial de los ecosistemas naturales de una región formando un continuo que aborda por completo el área del Distrito (Remolina 2011). Esta red de espacios produce bienes y servicios ambientales para la ciudad, siendo un proyecto prioritario para la Gestión Ambiental de Bogotá (Remolina 2007). Sin embargo, actualmente la EED presenta varias de sus áreas con un alto grado de intervención, lo que afecta su conectividad y genera una modificación en la estructura y composición del paisaje especialmente hacia las zonas rurales de Usme (Isaacs y Jaimes 2015).

A pesar de ello, el paisaje ha mantenido algunos remanentes de vegetación natural hacia las zonas de Cerros Orientales, Usme y Sumapaz, que debido a sus atributos de tamaño y forma, mantienen los flujos ecológicos y sus servicios ecosistémicos, ejerciendo una importante función conectora entre fragmentos (Remolina 2007, Correa 2008, Phillips y Navarrete 2009, Sguerra et al. 2011, Isaacs y Jaimes 2015). Este es el caso de las microcuencas Curubital, Mugroso, Chisacá y Regadera del Distrito Capital de Bogotá, las cuales presentan varios elementos de la EED, pero la conectividad no es continua con relación a las demás áreas protegidas como los bosques de los Cerros Orientales y a áreas de importancia nacional como el Parque Nacional Natural Sumapaz. Asimismo, es lugar de nacimiento de varios acueductos y ríos importantes, presenta invasión por retamo espinoso, plantaciones de pino y eucalipto y corresponde a una transición entre los bosques y el páramo, perteneciendo al complejo de páramos Cruz Verde - Sumapaz (Morales et al. 2007) y del corredor de conservación Sumapaz Chingaza - Guerrero. Estos atributos hacen la zona interesante para iniciar actividades de restauración, rehabilitación, recuperación y preservación, tema sobre el cual existen pocos trabajos desarrollados desde el análisis espacial para el Distrito y que son necesarios para definir las acciones específicas a tomar.

En el presente trabajo se buscó realizar una zonificación de áreas para la conectividad ecológica desde un análisis físico, biótico y social en las microcuencas nombradas, como insumo para definir áreas de restauración y preservación y que complementen las estrategias de conservación planteadas para esta zona.

\section{Material y métodos}

\section{Área de estudio}

La zona de estudio se encuentra ubicada en las microcuencas Curubital, Mugroso, Chisacá y Regadera, en la localidad de Usme, aunque toma algunas partes de la localidad de Sumapaz y Ciudad Bolívar al sur del Distrito Capital de Bogotá, Colombia (Figura 1). Las cuatro microcuencas se ubican sobre la cordillera Oriental y hacen parte de la gran cuenca del río Tunjuelo cubriendo un área de 11160 ha a una 
altura entre los 3.000 y 3.900 m s.n.m. (Isaacs 2011). De acuerdo al mapa de coberturas (Isaacs y Jaimes 2015), la zona está dominada por páramos y mosaicos de pastos y cultivos, en donde la frontera agrícola poco a poco ingresa a los páramos y solo mantiene algunos corredores riparios de matorrales transicionales, lo que ha venido amenazando la conservación de la zona (Isaacs 2011).

\section{Metodología}

Con base en la cartografía existente, salidas de verificación en campoy diferentes reuniones realizadas con expertos, se definieron tres criterios identificados como importantes en la selección de áreas para la zonificación espacial de la zona. Estos tres criterios se denominaron: 1) biótico, 2) socioeconómico y 3) físico, para construir un modelo de resistencia que sirviera de base para modelar conectividad. En estos términos, se tomó la conectividad como aquellas zonas que facilitan el movimiento de las especies y le dan continuidad al paisaje, con base en una grilla de resistencia y que conectan dos parches de hábitat los cuales se definieron con base en dos áreas protegidas que presentan la categoría de protección más alta en la EED (Adriaensen et al. 2003, Beier et al. 2008, McRae et al. 2008).

De acuerdo a la cartografía disponible para la zona, cada tipo de mapa fue asignado a uno de los criterios y se le definieron unas variables con base en los atributos que presentaba cada archivo. A cada uno de los atributos se le asignó un valor en su categorización si son más resistentes o no de acuerdo a un organismo en particular. En este caso, esto se hizo con el fin de que el modelo considerara aspectos funcionales para un usuario específico, en el que esos valores se ajustaban a los requerimientos de hábitat y necesidades de conservación del zorro común (Cerdocyon thous) que habita en la región y que fue evaluado según información secundaria de los registros de presencia para la zona reportados en bases de datos (ver Isaacs 2011). En este caso se consultó su rango de hogar y requerimientos de hábitat en la base de datos del Instituto de Ciencias de la Universidad Nacional (ICN), en la página de la UICN y en el libro de Mamíferos del Neotrópico de Emmons (1990). De la página del ICN, se descargaron los archivos de coordenadas para las especies, para conocer qué zonas son las que frecuenta y las que evita la especie.

\section{Criterio físico}

De acuerdo a lo anterior, para el criterio físico (Figura 2), se emplearon las capas de vías, ríos y cobertura. En primer lugar, las vías se encuentran categorizadas en tres niveles de acuerdo al tráfico y amplitud, a cada vía se le calculó un buffer de $100 \mathrm{~m}$ de incidencia y se le otorgó un valor, siendo más bajo para las de primer orden que son las más concurridas y por tanto más resistentes y hostiles para las especies. La justificación de los valores asignados se observan en la tabla 1. En segundo lugar, se incluyó la distancia a los ríos, a través de un buffer de $30 \mathrm{~m}$ y $100 \mathrm{~m}$ de ronda de protección de acuerdo a su magnitud (si son de primer, segundo o tercer orden), según lo propuesto por el decreto Decreto 2811 de 1974. Estos están subdivididos considerando que los ríos de primer orden tienen valores más altos de prioridad de conservación y seguido a estos se incluyeron las quebradas y cuerpos de agua. Por último, para este criterio se incluyó la capa de cobertura de la tierra, usando una clasificación manual de una imagen Ultracam (Igac 2010, escala 1:25.000). Para esta capa se asignaron mayores valores para las coberturas naturales y menor valor para las áreas intervenidas (Tabla 1).

Para el segundo criterio (socioeconómico), se usaron las áreas abastecedoras del recurso hídrico, tipo de uso del suelo potencial, capas de la EED y tamaño de predios. Se incluyeron las áreas abastecedoras de acueductos como zonas de protección del recurso hídrico y abastecimiento para los pobladores, lo cual es una razón de peso para las comunidades locales (Tabla 1). El tipo de uso del suelo es una propuesta que define cual es el uso más apropiado de una zona de acuerdo a sus características de suelo, relieve, vulnerabilidad y amenaza. Según esto, se clasifica en áreas donde el uso puede ser urbano y agrícola (el que mayor resistencia presenta), o el uso no es permitido y el área debe ser conservada (la resistencia es menor). 


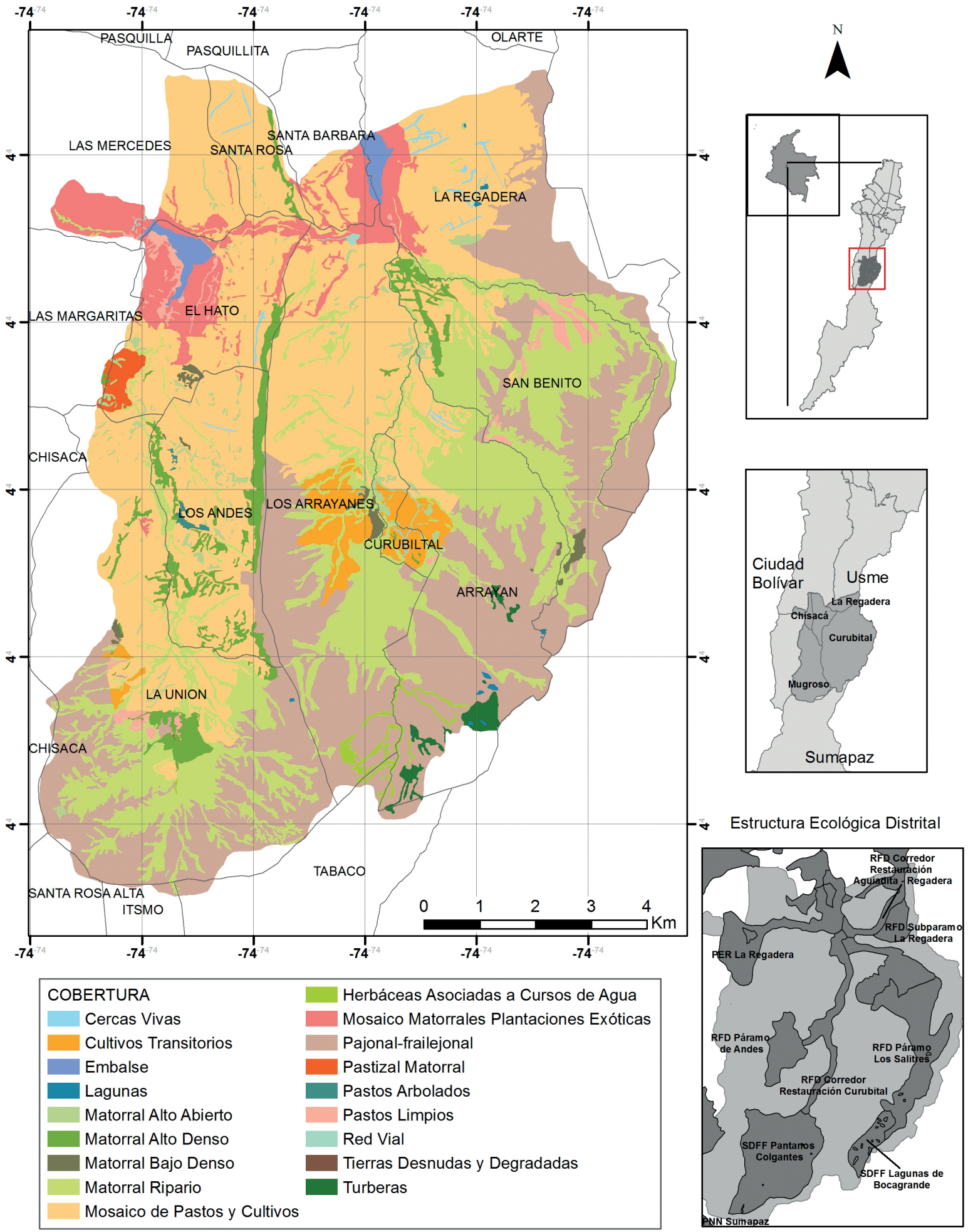

Figura 1. Cobertura de la tierra para las microcuencas Curubital, Mugroso, Chisacá y Regadera del Distrito Capital a escala 1:25.000 y su ubicación relativa. Elaboración propia, fuentes: cartografía SDA (2011), Igac (2014). 


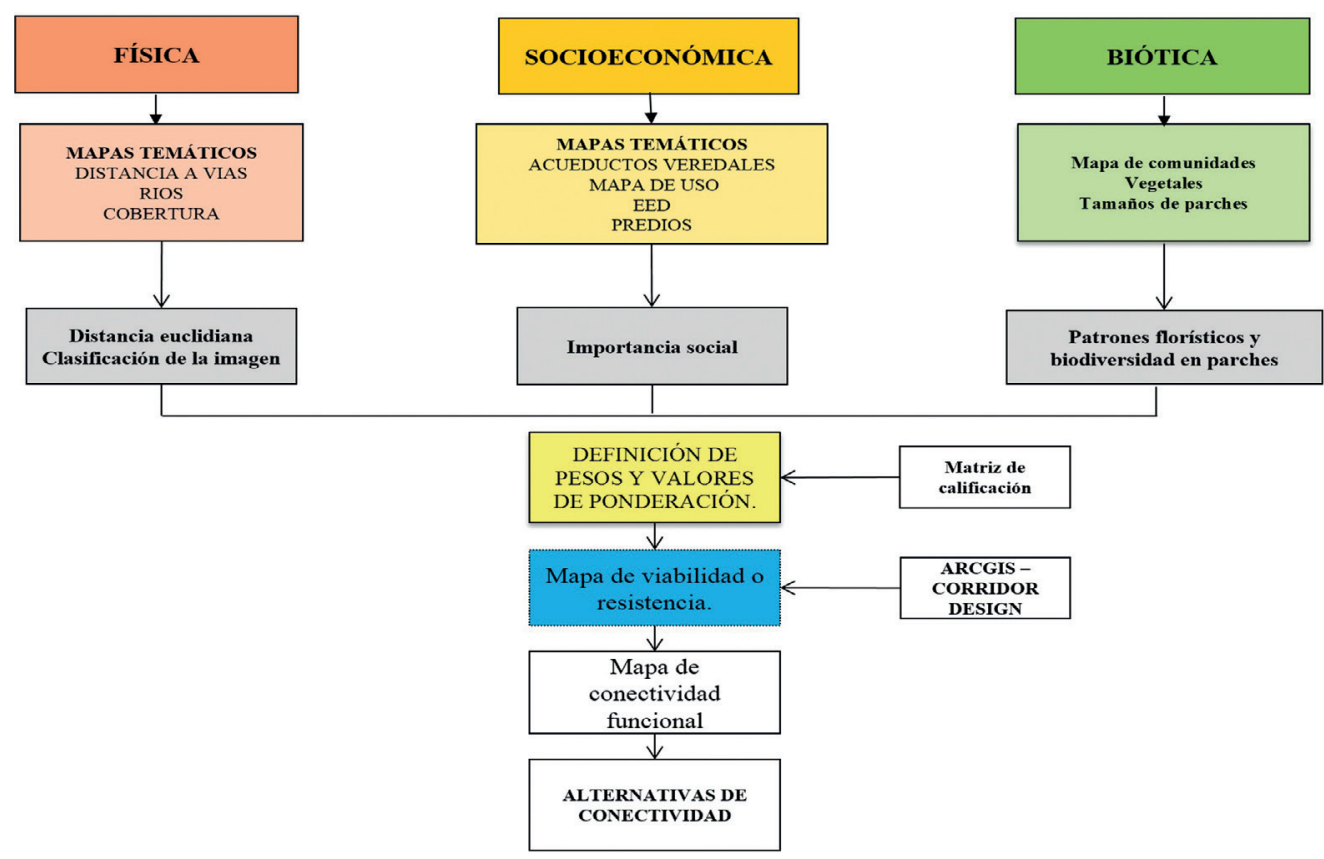

Figura 2. Metodología para el desarrollo de la evaluación y propuesta de conectividad.

\section{Criterio socioeconómico}

En este caso a pesar de tener un uso propuesto, no descarta que se deban realizar acciones de restauración. Para la EED presenta unas categorías de preservación y restauración previamente establecidas, en las que varía el grado de conservación o uso permitido. Especialmente el PNN Sumapáz y las Reservas Forestales Protectoras son las tierras donde hay mayor garantía de conservación a largo plazo debido a su categoría de protección, por eso recibieron los mayores valores. A las que presentan mayor flexibilidad en el uso permitido se les asignó menor valor ya que presentan menor estado de conservación a largo plazo. Finalmente, los tamaños de predio se tomaron para conocer la situación de la tenencia de la tierra en la zona y determinar las viabilidades sociales de la implantación ya sea de actividades de restauración o de conservación (Theobald 2006).

\section{Criterio biótico}

Finalmente, para el tercer criterio biótico, se incluyeron patrones florísticos y de la diversidad de las comunidades vegetales caracterizadas en estas microcuencas, en zonas donde se presenta la menor intervención posible según lo observado en los mapas de cobertura. Esto se incluyó con el fin de considerar aspectos bióticos para la conectividad adicionales a los reportes del zorro común, con base en esas zonas donde hay mayores condiciones de conservación para las especies y son más viables en términos también de hábitat. Adicionalmente, son zonas que presentan mayor valor por su riqueza, abundancia, diversidad, estructura y amenaza, como zonas de referencia y fuente para acciones de restauración (Trujillo 2012).

De acuerdo a esto, se contó con un total de 41 levantamientos realizados, para 15 comunidades vegetales en coberturas de bosques, bosques riparios, matorrales y páramos distribuidos en la zona de estudio. Con base en los levantamientos, se consideraron dos grandes atributos, uno relacionado con la diversidad que incluyó el índice de diversidad de Shannon, Índice de riqueza de Margalef, Diversidad Beta y Diversidad alfa y otro relacionado con los patrones florísticos que se establece para cada especie dentro de cada comunidad, incluyendo el Índice de Valor de Importancia - IVI, Índice de Predominio Fisionómico - IPF, Estado de amenaza y grado de exclusividad (Tabla 2). 
Tabla 1. Matriz de pesos construida para los diferentes criterios y variables a considerar para el modelo de conectividad propuesto. Los valores altos corresponden a zonas con menor resistencia y más viables para la conectividad.

\begin{tabular}{|c|c|c|}
\hline \multicolumn{3}{|r|}{ Variable de contectividad } \\
\hline Variable & Valor & Justificación \\
\hline Criterio físico & & Vías (Catastro distrital 2012) \\
\hline Vías primer orden & 0 & \multirow{3}{*}{$\begin{array}{l}\text { Las vías producen ruido y vibraciones que interfieren con las activida- } \\
\text { des de los individuos, promueven la erosión, crean barreras, fragmen- } \\
\text { tan el paisaje y favorecen la contaminación del agua (Beier et al. 2008, } \\
\text { Majka et al. 2009). Si su magnitud es mayor representa mayor riesgo } \\
\text { para las especies. }\end{array}$} \\
\hline Vías segundo orden & 3 & \\
\hline Vías tercer orden & 7 & \\
\hline \multicolumn{3}{|r|}{ Distancia a ríos (SDA 2012) } \\
\hline Ríos principales & 20 & \multirow{3}{*}{$\begin{array}{l}\text { La distancia a fuentes de agua, es un elemento de importancia para las } \\
\text { especies y la prestación de bienes y servicios ambientales (Beier et al. } \\
\text { 2008). Es eje primordial dentro de las políticas del Distrito para garan- } \\
\text { tizar el suministro de agua y las zonas riparias se constituyen como los } \\
\text { únicos remanentes de vegetación nativa. Los ríos principales presentan } \\
\text { mayor prioridad de conservación y por eso presentan mayor valor } \\
\text { (Jongman 2004). }\end{array}$} \\
\hline Quebradas & 10 & \\
\hline Cuerpos de agua & 5 & \\
\hline \multicolumn{3}{|c|}{ Cobertura de la tierra (JBB 2012) } \\
\hline $\begin{array}{l}\text { Herbáceas asociadas a cuerpos de } \\
\text { agua }\end{array}$ & 80 & \multirow{9}{*}{$\begin{array}{l}\text { La cobertura de la tierra es una variable de gran importancia dentro de } \\
\text { los modelos de hábitat de varias especies, reflejada en el hecho que está } \\
\text { relacionada con la alimentación, sitios de refugio e intervención antró- } \\
\text { pica (Beier et al. 2008, Majka et al. 2009). En este caso, las coberturas } \\
\text { de bosques y páramos son de gran importancia para la conservación } \\
\text { en el Distrito, ya que proveen gran cantidad de servicios ecosistémicos } \\
\text { y para la biodiversidad, especialmente lo que corresponde al recurso } \\
\text { hídrico y las poblaciones de flora y fauna nativa que aún se mantienen. } \\
\text { Los cultivos si bien eliminan la vegetación original, aún son fuente de } \\
\text { refugio y recursos para las especies de fauna y permiten procesos natu- } \\
\text { rales, a diferencia de los pastos para ganadería que con un mal manejo } \\
\text { deterioran los procesos y servicios de los ecosistemas (compactación y } \\
\text { degradación del suelo, afectación del drenaje, eliminación de la cober- } \\
\text { tura, entre otras; Jarro 2005). Las plantaciones exóticas mantienen la } \\
\text { - estructura de los bosques y permiten la presencia de fauna no siendo } \\
\text { tan hostiles como aquellas que eliminan la vegetación natural. Bajo } \\
\text { este orden de ideas, su cuantificación se asignó de mayor a menor valor } \\
\text { para la conservación. }\end{array}$} \\
\hline $\begin{array}{l}\text { Matorral ripario, alto denso y bajo } \\
\text { denso }\end{array}$ & 80 & \\
\hline Pajonal Frailejonal y Turberas & 80 & \\
\hline Matorral alto abierto & 15 & \\
\hline Pastizal matorral & 15 & \\
\hline $\begin{array}{l}\text { Mosaico plantaciones exóticas, } \\
\text { pastos arbolados y cercas vivas }\end{array}$ & 15 & \\
\hline Cultivos transitorios & 5 & \\
\hline Cuerpos de agua y lagunas & 5 & \\
\hline $\begin{array}{l}\text { Mosaico de pastos y cultivos, } \\
\text { pastos limpios y tierras desnudas y } \\
\text { degradadas }\end{array}$ & 0 & \\
\hline
\end{tabular}


Cont. Tabla 1. Matriz de pesos construida para los diferentes criterios y variables a considerar para el modelo de conectividad propuesto. Los valores altos corresponden a zonas con menor resistencia y más viables para la conectividad.

\begin{tabular}{ccc}
\hline Variable & Variable de contectividad \\
\hline Criterio socioeconómico & Justificación \\
\hline $0-10$ & 10 & $\begin{array}{c}\text { Tamaño de predio (ha.; Catastro 2012) } \\
\text { Se da prioridad a predios grandes con un solo propietario que permi- } \\
\text { tirán actividades y propuestas de manejo de forma más fácil que tratar } \\
\text { de concertar con muchos predios pequeños (Theobald 2006); Asimis- } \\
\text { mo, los predios mayores a 1000 ha presentan coberturas naturales de } \\
\text { mayor tamaño. }\end{array}$ \\
\hline $10-100$ & 60 &
\end{tabular}

Tipo uso del suelo (SDA 2012)

Sistema de Áreas Protegidas,

Sustracción de la reserva, zona a

alinderar por la CAR.

\begin{tabular}{lc} 
Manejo especial & 25 \\
\hline Alta fragilidad & 15
\end{tabular}

Alta capacidad 5

Aquellos suelos de expansión, de alta capacidad y con asentamientos menores tienen potencial de uso, así que no garantizan la conservación y tendrán menor posibilidad de implementación que aquellos de manejo especial, áreas de reserva y zonas a alinderar por la CAR que si tienen intrínseca la conservación sin proyección de usos y que no permiten actividades extractivas.

\begin{tabular}{ll} 
Asentamientos menores & 3 \\
\hline Suelo urbano y de expansión & 2
\end{tabular}

\section{Áreas abastecedoras de acueductos}

Áreas abastecedoras de acueductos

\section{0}

Preservación del recurso hídrico para los habitantes.

\section{Áreas protegidas - EEP}

\section{Parque Nacional Natural}

\begin{tabular}{ll}
\hline Reserva Forestal Protectora & 20 \\
\hline
\end{tabular}

Santuario Distrital de Fauna y Flora

15

Ronda Bogotá, Área forestal distrital, Parque ecológico distrital de humedal y PED de montaña
Áreas protegidas bajo un marco legal que garantiza la conservación a largo plazo de las zonas. Su calificación es mayor si la figura de protección es más fuerte. 
Cada criterio presentó una categoría de mayor a menor valor, a la cual se le asignó un valor de uno a cinco (Tabla 3). Con ello se construyó una matriz de valores acumulados por comunidad levantada, donde la ubicación de cada comunidad se asoció a un parche dentro del mapa de coberturas. Cabe aclarar que para el valor de los patrones florísticos, se suman los valores presentados por cada una de las especies presentes en cada comunidad.

Asimismo, estas coberturas naturales fueron reclasificadas por tamaños (Tabla 4), con el fin de priorizar aquellas zonas que tienen mayor potencial de conservación por su extensión y aquellas menos viables por su reducido tamaño. Esta variable de tamaño se ha reportado como una de las más importantes dentro de la evaluación de patrones del paisaje, por su relación con la mayor disponibilidad de hábitat para las especies, mayor funciones de la biodiversidad, reducción de endogamia, incidencia de efecto de borde, etc. (Goulart da Silva et al. 2015).

\section{Modelamiento de conectividad}

Posteriormente a la definición de los criterios y los valores de las variables de cada uno de los modelos, se obtuvieron los mapas de resistencia para cada criterio (físico, socioeconómico y biótico), el cual es el insumo para definir zonas de conectividad y áreas para restauración. Este muestra esas zonas en mejor estado de conservación y menor resistencia para las especies, que de acuerdo a los mapas obtenidos, corresponden a los mayores valores. Finalmente, se obtuvo un modelo que reúne la información de los tres criterios, en donde se identifican las áreas más viables que son útiles para plantear estrategias de conectividad y conservación, las más intervenidas y con mayor resistencia en donde se deberían plantear acciones de restauración según las condiciones de cada lugar. Esto se realizó mediante operaciones de algebra booleana de mapas para cada criterio usando ArcGIS 10.3. Por último, este modelo fue usado como insumo para modelar la conectividad mediante el uso del programa Circuitscape 4.0 (McRae et al. 2008). En este caso, se debe asignar dos zonas núcleo a conectar, las cuales por un lado debe tener mejor estado de conservación, deben ser áreas protegidas declaradas y su categoría de protección no debe ser amenazada a futuro y siendo lo más rigurosa posible. De acuerdo a esto, se tomó como núcleos dos áreas ubicadas en los extremos de las microcuencas como el Corredor de conservación, Aguadita - La Regadera y el Parque Nacional Natural Sumapaz.

Tabla 2. Criterios planteados para la evaluación de las comunidades vegetales para el criterio biótico.

\begin{tabular}{lll}
\hline \multicolumn{2}{c}{ Criterio de Evaluación } & \multicolumn{1}{c}{ Concepto } \\
\hline \multirow{3}{*}{$\begin{array}{l}\text { Indicadores de } \\
\text { Biodiversidad }\end{array}$} & Diversidad de Shannon & Diversidad de especies en la comunidad \\
\cline { 2 - 3 } & Riqueza de Margalef & Número de especies en la comunidad \\
\cline { 2 - 3 } & Diversidad Beta & Diversidad de especies a nivel regional \\
\cline { 2 - 3 } Patrones florísticos & Diversidad Alfa & Diversidad de especies a nivel local \\
\cline { 2 - 3 } & IVI & Índice de Valor de Importancia \\
\cline { 2 - 3 } & Estado de amenaza & Índice de Predominio Fisionómico \\
\cline { 2 - 3 } & Grado de exclusividad & $\begin{array}{l}\text { Categoría de Amenaza } \\
\text { sitio de estudio. }\end{array}$ \\
\hline
\end{tabular}


Tabla 3. Categorización de los valores asignados a cada uno de los criterios para el criterio biótico.

\begin{tabular}{lll}
\hline \multicolumn{1}{c}{ Criterio } & \multicolumn{1}{c}{ Categoría } & Valor \\
\hline Diversidad & Muy alto & 5 \\
\hline Riqueza & Alto & 4 \\
\hline Abundancia & Medio & 3 \\
\hline IVI & Bajo & 2 \\
\hline \multirow{2}{*}{ IPF } & Muy Bajo & 1 \\
\hline \multirow{4}{*}{ Estado de amenaza } & En Peligro Crítico (CR) & 5 \\
\cline { 2 - 3 } & En Peligro (EN) & 4 \\
\cline { 2 - 3 } & Vulnerable (VU) & 3 \\
\cline { 2 - 3 } & Casi Amenazada (NT) & 2 \\
\cline { 2 - 3 } & $\begin{array}{l}\text { Preocupación Menor } \\
\text { (LC) }\end{array}$ & 1 \\
\cline { 2 - 3 } & Datos Insuficiente (DD) & 0 \\
\hline \multirow{3}{*}{$\begin{array}{l}\text { Grado de } \\
\text { exclusividad }\end{array}$} & $\begin{array}{l}\text { Endémica páramos de } \\
\text { Colombia }\end{array}$ & 5 \\
\cline { 2 - 3 } & Endémica Colombia & 5 \\
\cline { 2 - 3 } & Restringida a páramo & 3 \\
\cline { 2 - 3 } & Restringida a bosque & 3 \\
\cline { 2 - 3 } & Amplia distribución & 0 \\
\hline & & \\
\hline
\end{tabular}

Tabla 4. Categorización de los tamaños de parche y su valor asignado para el criterio biótico.

\begin{tabular}{cc}
\hline $\begin{array}{c}\text { Tamaños de parches } \\
\text { vegetación }\end{array}$ & Valor \\
\hline $0-10$ ha & 5 \\
\hline $11-100$ ha & 15 \\
\hline $101-999$ ha & 30 \\
\hline$>1000$ ha & 50 \\
\hline
\end{tabular}




\section{Resultados}

\section{Modelo físico}

Como resultado de la modelación para el primer criterio, se encuentran en rojo las áreas que representan un hábitat más hostil para las especies. En este caso las zonas menos favorables son aquellas pertenecientes a cultivos y pastos en las que se busca evitar vías y favorecer la conservación de los drenajes. En verde, se encuentran las zonas de coberturas naturales de páramos y matorrales y se destacan las zonas de drenajes especialmente, también se pueden observar pequeñas zonas intervenidas en color rojo en la vereda San Benito y al sur de la vereda La Unión (río Mugroso). Con valores intermedios en naranja y amarillo se encuentran las áreas de especies exóticas de los embalses de La Regadera y Chisacá (Figura 3).

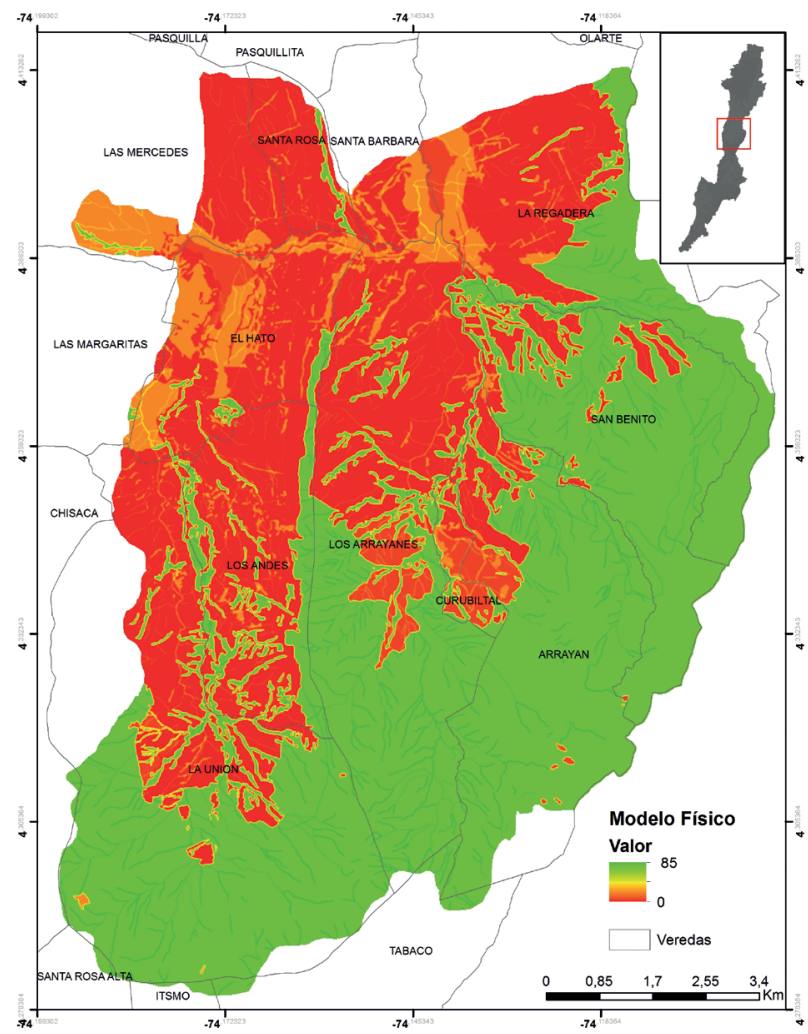

Figura 3. Modelo resistencia generado con las capas de cobertura, distancia a ríos y vías según el primer criterio físico. Escala (1:25.000). En rojo los valores con mayor resistencia y en verde los más viables para las especies.

\section{Modelo socioeconómico}

Por su parte el segundo modelo (criterio socioeconómico) se ve altamente influenciado por el tamaño de los predios y de las áreas protegidas declaradas de la EED, las cuales son de gran tamaño y que son las que se encuentran en verde; en amarillos se encuentran las áreas de manejo especial y en naranja las áreas de usos restringidos (Figura 4). Las demás divisiones al interior responden a los usos del suelo permitidos.

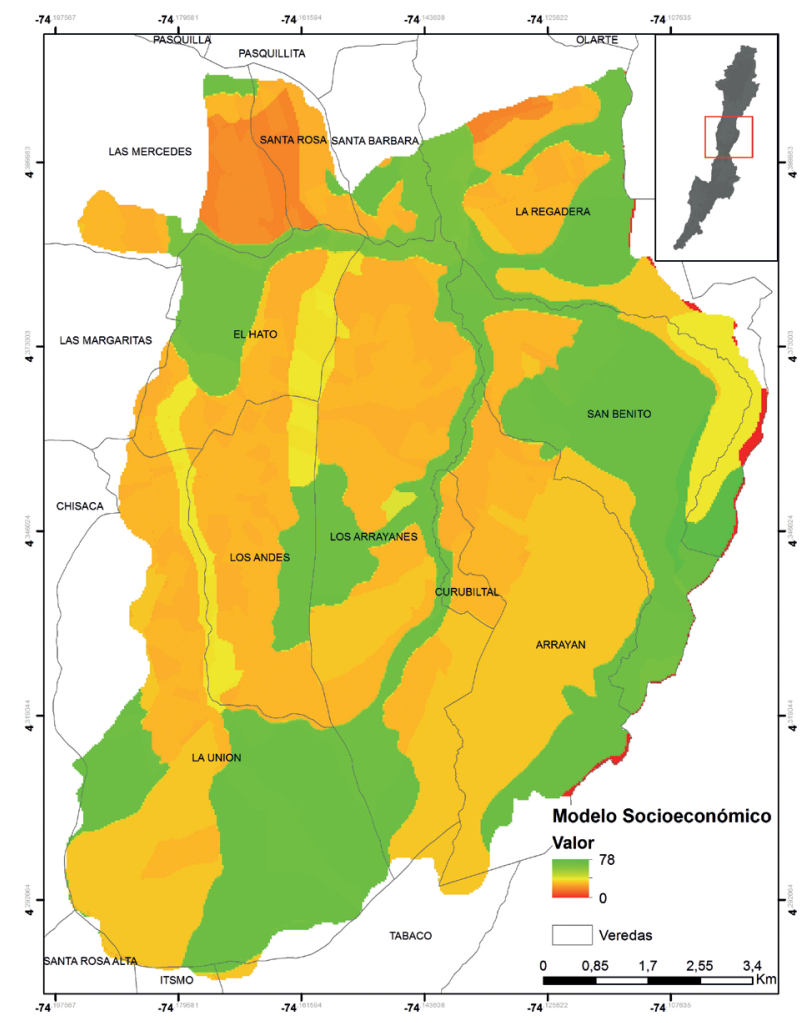

Figura 4. Segundo modelo de resistencia generado con las capas de EED, tamaño de predios, uso del suelo y áreas abastecedoras del recurso hídrico para el criterio socioeconómico. Escala 1:25.000. En rojo los valores con mayor resistencia y en verde los más viables para las especies. 


\section{Modelo biótico}

Para el tercer modelo, de la cuantificación asignada con base en los datos de campo, se observa una zona de alta conservación al sur de la vereda los Arrayanes, una zona intermedia en amarillo y una naranja entre las veredas Los Arrayanes, Arrayán, Curubital y San Benito (Figura 5). En estas zonas los mayores valores se presentaron en los matorrales riparios donde se presentan comunidades dominadas por Bucquetia glutinosa - Gaultheria anastomosans - Diplostephium rosmarinifolium Macleania rupestris - Befaria resinosa, Espeletia grandiflora - Arcytophyllum nitidum -Vaccinium floribundum - Weinmannia tomentosa - Ilex kunthiana - Valea stipularis. Estas zonas coinciden con áreas de mayor diversidad y abundancia, así como valores altos de IVI e IPF. Por su parte, en rojo se observan aquellas zonas intervenidas, o donde las comunidades presentan valores bajos según los patrones florísticos y la diversidad.

De igual manera, los matorrales altos se destaca la comunidad dominada por I. kunthiana, M. ligustrina, $M$. rupestris, aunque se establecieron comunidades en donde la especie dominante es $W$. fagaroides o $W$. tomentosa con menor importancia. Estas últimas especies consideradas importantes para el mantenimiento de las coberturas de bosque, presentan un reducido o ausente sotobosque, siendo unas pocas especies las que dominan. Por su parte, los matorrales riparios al sur de la microcuenca Curubital presentan comunidades dominadas por $B$. glutinosa - G. anastomosans - D. rosmarinifolium M. rupestris - B. resinosa, E. grandiflora - A. nitidum $-V$. floribundum - W. tomentosa - I. kunthiana - V. stipularis, con el menor valor de resistencia reportada.

Como modelo resultante de los tres criterios se obtuvo un mapa de resistencia con la zonificación que presenta áreas por donde sería más viable plantear conectividad ecológica, así como las áreas más conservadas con mejores coberturas y estructura vegetales, que presentan una figura de protección más alta en la EED y un uso de la tierra restringido (Figura 6). En amarillo se encuentra las zonas que a pesar de tener cobertura natural no presentan categorías de protección de la EED y tienen un valor asignado por su importancia en términos de la vegetación. En naranja están aquellas zonas con una medida de protección más laxa y uso permitido, con presencia de pastos y cultivos. Y en rojo aquellas zonas que no presentan protección, ausencia de vegetación natural y usos permitidos (Figura 6).

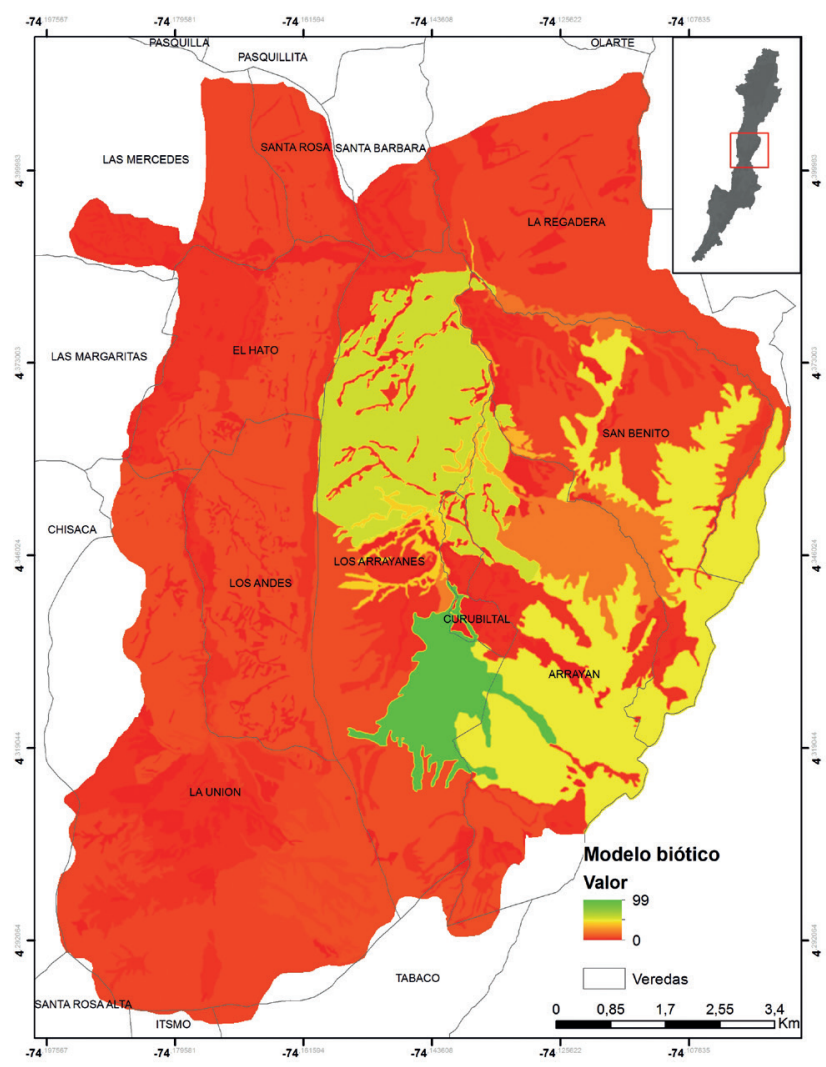

Figura 5. Tercer modelo de resistencia integrando los levantamientos de las comunidades vegetales. Escala 1:25.000. En rojo los valores con mayor resistencia y en verde los más viables para las especies. 


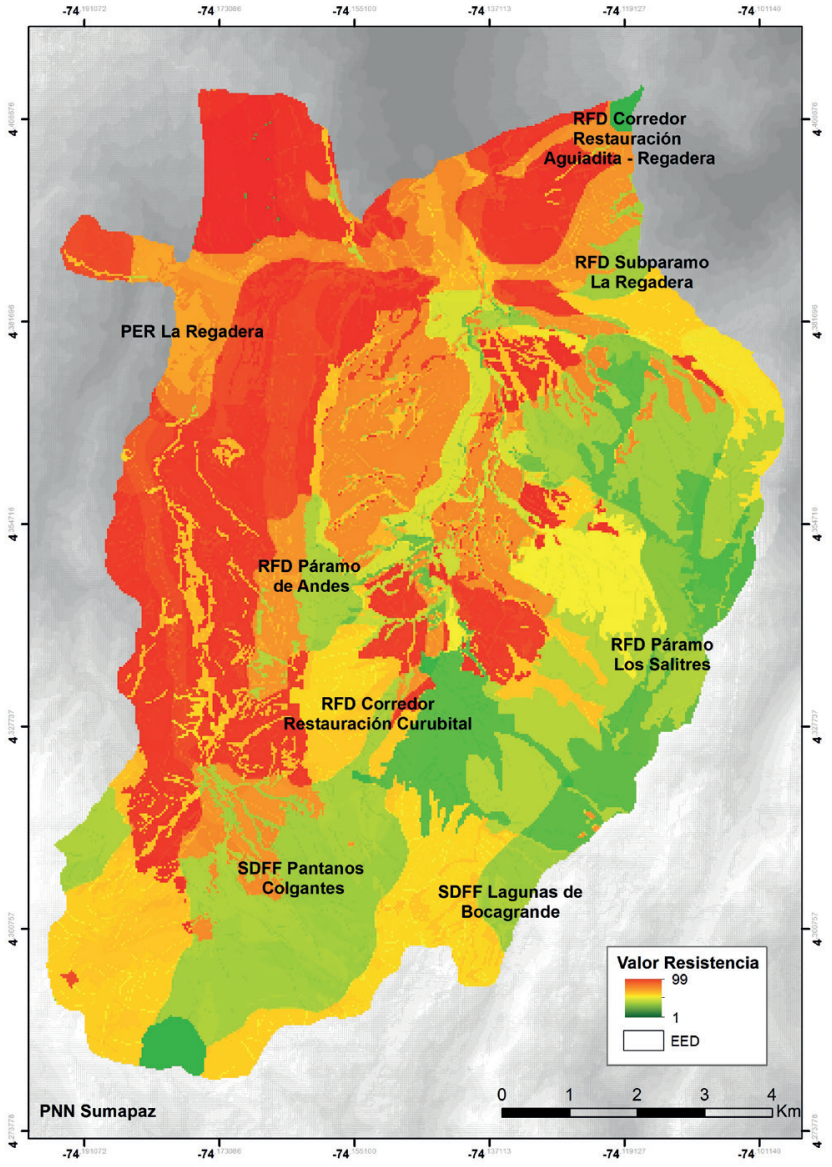

Figura 6. Modelo resultante de la combinación de los tres criterios para el área de estudio. En rojo los valores con mayor resistencia y en verde los más viables para las especies.

Por último, el modelo de conectividad obtenido muestra las zonas con mejor estado de conexión para las microcuencas evaluadas, especialmente sobre las zonas con cobertura natural sobre las áreas de la EED. El modelo adicionalmente muestra las zonas con menor valor de conectividad con coberturas antrópicas y que muestra otras zonas diferentes a la EED (Figura 7).

\section{Discusión}

Elobjetivo del presente trabajo buscó plantear modelos de conectividad que se centraran en ubicar áreas que faciliten el movimiento de las especies mediante la identificación de áreas conservadas y en aquellas que se centraran en restaurar la conectividad en zonas que presenten intervención o una figura de protección más laxa o ausente. Se buscó adicionalmente generar un modelo metodológico en la escogencia de criterios y de variables que identificaran zonas de resistencia o permeabilidad, como base para la planificación y el diseño de acciones de conservación (entendiendo conservación como el conjunto de acciones de preservación, restauración y uso sostenible).

La base de estos modelos se ha construido de acuerdo a diferentes enfoques disponibles como las grillas de menor costo (Beier et al. 2008), teoría de circuitos (McRae et al. 2008), modelos con base en

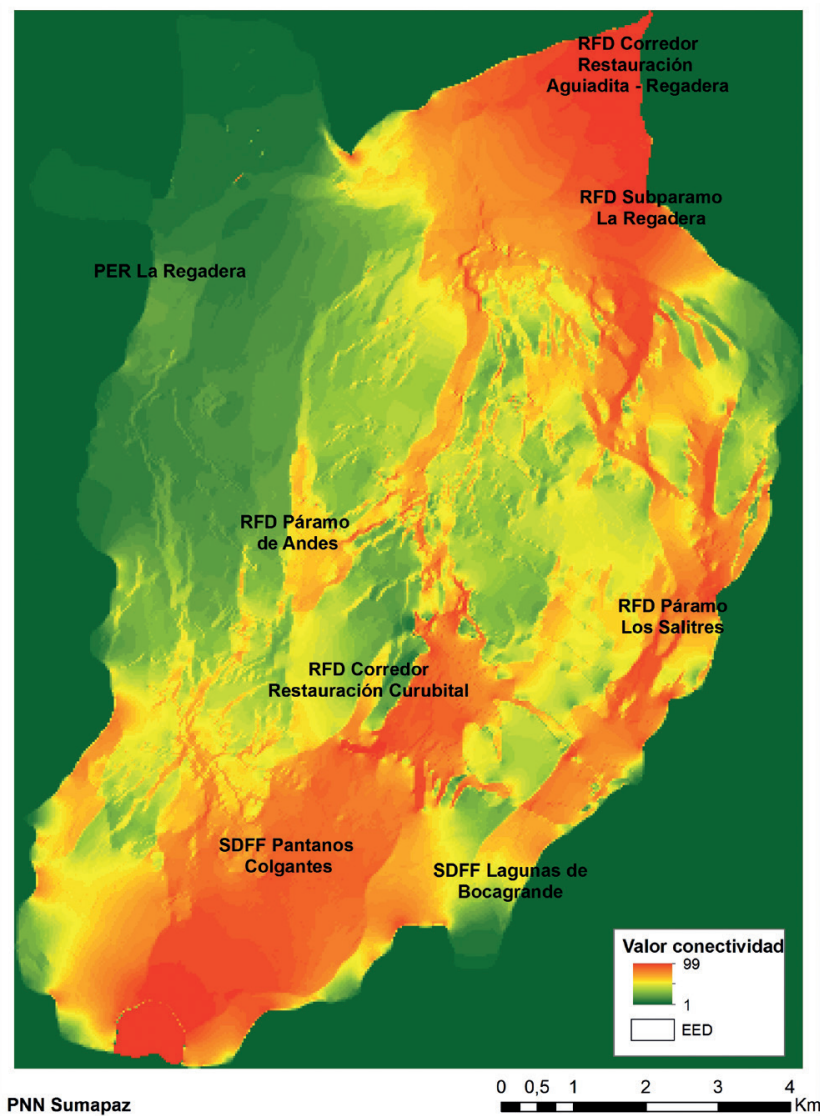

Figura 7. Modelo de conectividad obtenido de los criterios empleados. En rojo se presenta la red de conectividad, la cual corresponde a los valores con mayor viabilidad para implementar y mantener estrategias de conectividad. Los valores con mayor resistencia en verde, muestran aquellas zonas en donde se deben implementar estrategias de restauración (áreas intervenidas) y manejo del paisaje (áreas de páramo y zonas sin categorías de protección). 
movimiento (Tracey et al. 2006) entre otros, que no modelan únicamente corredores como líneas en el paisaje, sino que permiten identificar áreas con potencial de conservación y áreas con posibilidades de restauración u otros tipos de manejo del territorio que abran acciones potenciales para mejorar la conectividad (McRae et al. 2012)

Estos mapas deben ser interpretados por los tomadores de decisión a la luz de costos, beneficios y otros objetivos de manejo, sin embargo, estas herramientas son el insumo base para la planeación de las estrategias de restauración del paisaje y con una perspectiva de paisaje (Metzger y Brancalion 2013, Tambosi et al. 2014, Isaacs y Ariza 2015).

La conectividad en el Distrito ha tenido diversos planteamientos para recrear, restaurar o fortalecer las uniones entre las áreas de la Estructura Ecológica Distrital (Sguerra et al. 2011, Remolina 2011). Desde su conformación, la EED ha buscado determinar la conectividad entre las coberturas que presenta el Distrito, con una gran necesidad de abordar criterios ecológicos en términos de movimientos locales, dispersión o migración de las especies y que a su vez sea cuantificada y representada en términos de indicadores espaciales que determinen las acciones de conservación a llevar a cabo en términos de restauración ecológica, rehabilitación, recuperación y preservación (Remolina 2011, MADS 2014).

De acuerdo a esto, se abordaron tres criterios que incluyen diferentes aspectos que permiten visualizar la configuración espacial de la zona de estudio. El primero de los modelos, muestra principalmente las zonas con vegetación nativa de páramos y matorrales, aunque es posible identificar áreas intervenidas, que no favorecen la conectividad y que deben ser evitadas. Así mismo, los matorrales riparios y páramos presentan menor resistencia debido a que en esas zonas se encuentran los parches más grandes lo que incrementa su diferencia. Con valores intermedios se presentaron aquellas zonas al borde entre el páramo y los matorrales, con áreas intervenidas donde aún hay matorrales altos y bajos abiertos que hacen una transición entre las coberturas naturales y los cultivos y pastos. Hacia las zonas de plantaciones los valores de resistencia son mayores por su tamaño y composición, la cual requiere de un manejo especial por corresponder a especies exóticas con algunas invasiones de retamo espinoso. Sin embargo, estas coberturas están rodeadas de una matriz de uso antrópico, aunque actualmente mantenga remanentes de vegetación importantes para la conservación como los matorrales riparios y algunas zonas con valor intermedio de plantaciones exóticas.

Igualmente para este modelo, se observa que en la zona no se han construido vías de gran magnitud, lo que no afecta la continuidad de las coberturas pero si son elementos que se deben evitar si se plantean corredores o zonas de conservación, por las vibraciones, accesibilidad de autos y personas y riesgo de atropellamiento de la fauna ( $\mathrm{Ng}$ et al. 2004). Adicionalmente es posible observar en este modelo, que las zonas de drenajes merecen una mayor importancia tanto para la preservación, como la restauración para un beneficio en términos de bienes y servicios ambientales, así como un insumo primordial para la conectividad de las especies (Phillips y Navarrete 2009, Remolina 2011), sin dejar de lado la importancia de manejar igualmente otras áreas que complementan la conservación de las zonas (Beier et al. 2008). Esto se ha planteado como dos de los elementos del paisaje que deben siempre abordarse dentro de los modelos de conectividad y en especial de conservación (Beier et al. 2008, Tambosi y Metzger 2014, Goulart da Silva et al. 2015).

Es importante destacar, que las zonas más hostiles cubiertas por pastos y cultivos en su momento pertenecieron a áreas transicionales de bosques altoandinos, por lo que se deben encaminar acciones de restauración y rehabilitación de este ecosistema y centrarse en reconectar las áreas naturales hacia los páramos, para garantizar la integridad de la zona. Igualmente, es preocupante el avance que están presentando los cultivos de papa hacia los páramos, con la recurrente incidencia de quemas para su cultivo y la escasa presencia de vegetación en las rondas de los cuerpos de agua (Isaacs y Jaimes 2015). 
El segundo modelo, dado que dio mayor peso a la EED, muestra menor resistencia, seguida de otras zonas de matorrales y páramos en naranja que tienen también una propuesta de uso del suelo más restrictivo. Este modelo mostraría áreas que podrían servir como zonas de amortiguamiento de la EED, en donde se pueden dar actividades productivas como sistemas silvopastoriles y agroforestales, que irían enriqueciendo el paisaje intentando devolver su funcionalidad y proveer actividades productivas a los pobladores (Funambiente 2009, Lozano 2014). De acuerdo a lo propuesto en la red de la EED, se debe considerar la categoría de protección que propone, así como el tipo de uso propuesto en las áreas circundantes, lo cual facilitaría la dirección en la que se deben encaminar las acciones de restauración.

Estas cuencas son áreas estratégicas para realizar acciones de restauración, ya que no existe un área dentro de la EED que este conservando coberturas de bosque y que este aportando a la representatividad de las mismas, todas dentro de su figura conservan páramos y subpáramos y únicamente el corredor de conservación del río Curubital estaría ocupando áreas más bajas en donde originalmente se encontraban bosques altoandinos (Sguerra et al. 2011, Isaacs y Jaimes 2015). La importancia de restaurar los bosques se debe a que no hay una continuidad entre los Cerros Orientales y las zonas de Sumapaz, por lo que esta fragmentación está afectando la continuidad de las coberturas naturales y la dispersión de los organismos que aún se presentan en el Distrito. Asimismo, el área de estudio hace parte del corredor de conservación Sumapaz, Chingaza, Guerrero, el cual propone el área como zona de conservación que complementa a su vez la Estructura Ecológica de la ciudad-región (Remolina 2011, Sguerra et al. 2011).

Para el tercer modelo, se destacan en cada comunidad aquellas que presentaron una mayor estructuración y complejidad, no siendo siempre las que presentaban las especies más representativas o los individuos más desarrollados, pero si son zonas con menor resistencia que actuarían como núcleos o sitios de paso de la conectividad (Tambosi y Metzger 2013, Tambosi et al. 2014).

Este mayor peso a aquellas comunidades con diversidad alta es de gran relevancia para una estrategia de restauración ecológica y preservación ya que se traduce en mayor disponibilidad de hábitat para las especies, en términos de cobertura y alimento. Igualmente, estas zonas podrían ser fuentes de semillas y plántulas para la restauración de las áreas circundantes, en donde se busca ir incrementando el tamaño de los parches progresivamente y de las pocas zonas riparias que se encuentran al norte de Los Arrayanes (Goulart da Silva et al. 2015). Estas zonas al presentar mejor estado de conservación de acuerdo a la composición de las comunidades, permitirían acciones de restauración de la vegetación nativa y estaría constituyéndose como una zona de restauración de bosque natural que complementa esa figura del corredor de restauración del río Curubital.

Como es de esperar, puede existir un sesgo debido a la cantidad de levantamientos de vegetación realizados y la extensión de cada cobertura, sin embargo, estas zonas corresponden a los parches más conservados y que contienen muestras importantes de la composición original de la zona. Como en el modelo se incluyeron tamaños de parches, adicionalmente se da peso a aquellos que más contribuyen a la conservación y que igualmente representan un gran aporte para la restauración.

La unión de los tres modelos, está construida con los tres criterios que se comportan como una grilla de resistencia y que muestra las zonas más conservadas para plantear conectividad (McRae et al. 2008). Se destaca el valor que es asignado a la EED; el parche de permeabilidad alta ubicado en la vereda Los Arrayanes que ocupa matorrales riparios y matorrales altos, es de gran importancia para fortalecer la EED, ya que es adyacente al corredor de restauración Curubital y no hace parte en su totalidad de esas áreas de conservación. En todos los modelos presenta un valor de permeabilidad alto no solo por su valor en términos de comunidades, sino en cuanto a cobertura, 
tamaño de parche y uso permitido. Esta zona podría complementar el Área del Santuario Distrital de Pantanos Colgantes, añadiendo representatividad de las coberturas de matorrales altos densos y riparios, ya que hasta el momento solo contiene coberturas de páramo (ver figura 6).

Para las demás áreas, el mapa final aporta insumos que orientan la decisión de áreas de conservación y restauración como se nombró anteriormente, así como aquellas por donde es más viable plantear conectividad, entendido como el conjunto de acciones tanto de restauración como de manejo en el caso de áreas que están preservadas. Las decisiones deben ser tomadas con información más detallada, obtenida en campo, que valide la propuesta presentada y que permita definir el tipo de restauración a llevar a cabo o la propuesta de áreas de preservación (AguilarGaravito e Isaacs 2014).

La escogencia de la EED, buscaba centrar la zonificación en las áreas protegidas consideradas como fuente y conservación de las especies, las cuales deben contar con un manejo adecuado que garantice a largo plazo su conservación ya que no tendrá ningún sentido conectar zonas que no proveerán los recursos necesarios para supervivencia de las especies (Gurrutxaga 2004, Hodgson et al. 2011).

Es importante destacar que el presente análisis se realizó únicamente para las zonas del Distrito, como respuesta a las necesidades de gestión en términos de división política, a pesar de considerar límites de microcuencas. Sin embargo, la conectividad y las zonas de resistencia se deben mirar desde un marco geográfico más amplio que responda de mejor forma a un modelo netamente biótico y no limitado por variables antrópicas, a pesar que la valoración de los criterios siempre fue pensado en términos funcionales de la fauna, en este caso requerimientos de hábitat de los mamíferos y características de la flora (Fandiño y Wyngaarden 2003, Magioli et al. 2015). Esto busca propiciar espacios que faciliten la toma de decisiones coordinadas entre las autoridades ambientales que cuentan con áreas protegidas colindantes o que comparten las mismas dificultades u oportunidades de manejo, de forma que se logre mayor coherencia y efectividad en la gestión (Sguerra et al. 2011).

El rápido cambio y la severidad de las amenazas a la biodiversidad son tan fuertes especialmente en zonas como el Distrito Capital, que es urgente la necesidad de desarrollar lineamientos con base en criterios técnicos hacia la construcción de medidas efectivas para garantizar a largo plazo la protección de la biodiversidad. Aspectos como las ubicaciones más probables y las zonas en donde es más viable proponer acciones de conectividad, el tipo de especies que beneficiará y la forma de monitorear estos procesos, son los tipos de preguntas que deben buscar orientar estos modelos (Gurrutxaga 2004, Theobald 2006).

\section{Conclusiones}

El presente escrito se presenta como un modelo que permite visualizar una zonificación de las tres microcuencas evaluadas con el fin de priorizar áreas de conservación, restauración y aquellas que facilitan la conectividad entre las coberturas presentes en el diseño, planeación y monitoreo. El uso de diferentes criterios permite tener un panorama más amplio a la hora de tomar decisiones y en especial en el diseño de su implementación. Adicionalmente, el presente análisis es un insumo que complementa los diseños propuestos de áreas protegidas con base en la configuración y composición del paisaje en donde es posible identificar diferentes escenarios de conectividad modelados.

\section{Agradecimientos}

Los autores queremos agradecer la colaboración del equipo del Jardín Botánico de Bogotá, a las personas que apoyaron los levantamientos en campo y al equipo de la Subdirección Científica quien nos brindó sus comentarios y sugerencias en la mejora del trabajo y sus análisis. 


\section{Bibliografía}

Adriaensen, F., J. P. Chardon, G. de Blust, E. Swinnen, S. Villalba, H. Gulink y H. Matthinsen. 2003. The application of 'least-cost' modelling as a functional landscape model. Landscape and Urban Planning 64: 263-274.

Aguilar-Garavito, M. y P. Isaacs. El análisis espacial en la restauración ecológica. 2014. Capítulo 2.5. Pp. 95109. En: M. Cabrera y W. Ramírez (Eds.). Restauración ecológica de los páramos de Colombia. Transformación y herramientas para su conservación. Instituto de Investigación de Recursos Biológicos Alexander von Humboldt.

Beier, P., D. R. Majka y W. D. Spencer. 2008. Forks in the road: choices in procedures for designing wildland linkages. Conservation Biology 22: 836-851.

Bennett, A. F. 2006. Linkages in the landscape: role of corridors and connectivity in wildlife conservation. IUCN, Gland, Switzerland and Cambridge, UK. 254 pp.

Correa, C. 2008. Análisis del estado actual de conectividad de las coberturas vegetales de la cuenca media del río Tunjuelo. Jardín Botánico de Bogotá José Celestino Mutis. Revista Pérez Arbelaezia 12: 117-141.

Doak, D. F. 1995. Source-sink models and the problem of habitat degradation: general models and applications to the Yellowstone Grizzly. Conservation Biology 96: 1370-1379.

Emmons, L. 1990. Neotropical rainforest mammals: a field guide. University of Chicago. 396 pp.

Fandiño, M. y W. Wyngaarden. 2003. Rol de la fauna en la selección de áreas de conservación biológica -base conceptual y metodológica-. Pp: 205-209 En: PolancoOchoa, R. (Ed.). Manejo de fauna silvestre en amazonía y Latinoamérica. Selección de trabajos V Congreso Internacional. CITES, Fundación Natura. Bogotá, Colombia.

Forman, R. T. T. 1995. Land Mosaics: The ecology of landscapes and regions. Cambridge University Press. Cambridge, United Kingdom. 632 pp.

Funambiente. Corporación Salvemos el Medio Ambiente. 2010. Formulación de lineamientos ambientales de conectividad de la operación estratégica Nuevo Usme como propuesta piloto en el sector de borde urbano rural. Informe Técnico. $215 \mathrm{pp}$.

Goulart da Silva, L., M. C. Ribeiro, E. Hasui, C. Aparecida da Costa, R. Teixeira da Cunha. 2015. Patch size, functional isolation, visibility and matrix pemeability influences neotropical primate occurence within highly fragmented landscapes. Plosone 10: 1- 20.

Gurrutxaga San Vicente, M. 2004. Conectividad Ecológica del Territorio y Conservación de la Biodiversidad.
Nuevas Perspectivas en Ecología del Paisaje y Ordenación Territorial. Gobierno Vasco. 85 pp.

Hargrove. W. W., F. M. Hoffman y R. A. Efroymson. 2004. A Practical Map-Analysis Tool for Detecting Potential Dispersal Corridors. Landscape Ecology 20: 361-373.

Hodgson, J. A., C. D. Thomas, B. A. Wintle y A. Moilanen. 2009. Climate change, connectivity and conservation decision making: back to basics. Journal of Applied Ecology 46: 964-969.

Isaacs, P. J. 2011. Definición de las alternativas de conectividad ecológica posibles desde el análisis físico, biótico y espacial de las áreas rurales priorizadas por la subdirección científica. informe técnico contrato 334/2011. Jardín Botánico de Bogotá Jardín Botánico de Bogotá José Celestino Mutis. 80 pp.

Isaacs, P. J. y V. Jaimes. 2015. Análisis multitemporal de las coberturas del Distrito Capital años 1990 - 2012. Jardín Botánico de Bogotá José Celestino Mutis. 65 pp.

Jarro, C. 2005. Guía técnica para la restauración ecológica de áreas afectadas por la expansión agropecuaria en el Distrito Capital. Jardín Botánico de Bogotá José Celestino Mutis. Bogotá, Colombia. 155 pp.

Jongman, R., M. Kulvik e I. Kristiansen. 2004. European ecological networks and greenways. Landscape and Urban Planning 68: 305-319.

Lozano, F. 2014. Diseño de herramientas de manejo de paisaje como estrategia para la conservación y restauración en cuencas del departamento del Valle del Cauca. Informe técnico Convenio 13-166. Instituto de Investigación de Recursos Biológicos Alexander von Humboldt y CVC. 242 pp.

Magioli, M., M. C. Ribeiro, K. M. P. M. B. Ferraz y M. G. Rodríguez. 2015. Thresholds in the relationship between functional diversity and patch size for mammals in the Brazilian Atlantic Forest. Animal Conservation 1-13.

Majka, D., J. Jenness y P. Beier. 2009. CorridorDesigner: ArcGIS tools for designing and evaluating corridors. Available at http://corridordesign.org.

McRae, B. H., B. G. Dickson, T. H. Keitt, y V. B. Shah. 2008. Using circuit theory to model connectivity in ecology and conservation. Ecology 10: 2712-2724.

Medici, P., P. Mangini y J. A. Sarria. 2007. Manual de medicina veterinaria de antas em campo. IUCN/SSC TAPIR SPECIALIST GROUP (TSG). Comitê de Veterinária. Informe técnico. $60 \mathrm{pp}$.

Meffe, G. K. y C. R. Carroll. 1997. Principles of Conservation Biology. Sunderland, Massachusetts: Sinauer Associates. 779 pp.

Metzger, J. P. y P. Brancalion. 2013. Challenges and opportunities in applying a landscape ecology perspective 
in ecological restoration: a powerful approach to shape neolandscapes. Natureza \& Conservação 11: 103-107.

Ministerio de Ambiente y Desarrollo Sostenible - MADS. 2014. Plan Nacional de Restauración. Documento técnico. Consultado en línea Febrero 12 de 2015. 92 pp.

Morales, M., J. Otero, T. Van der Hammen, A. Torre, C. Cadena, C. Pedraza, N. Rodríguez, C. Franco, J. C. Betancourth, E. Olaya, E. Posada y L. Cárdenas. 2007. Atlas de páramos de Colombia. Instituto de Investigación de Recursos Biológicos Alexander von Humboldt. Bogotá, D. C. 208 pp.

Murcia, C. 1995. Edge effects in fragmented forest implications for conservation. Trends in Ecology and Evolution 10:58-62.

Ng, S., J. W. Dole, R. M. Sauvajot, S. Riley y T. J. Valone. 2004. Use of highway undercrossings by wildlife in Southern California. Biological Conservation 115: 499 -507 .

Noss, R. 1991. Landscape connectivity: Different functions at different scales. Capítulo 2. En: Hudson W. E. (Ed.). Landscape linkages and biodiversity. Defenders of Wildlife. Island Press. Washington D.C.

Noss, R. y A. Y. Cooperrider. 1994. Saving Natures Legacy: Protecting and restoring biodiversity. Defender of Wildlife. Island Press. Washington D. C. 417 pp.

Phillips, J. F y D. A. Navarrete. 2009. Análisis de fragmentación y conectividad. Informe Final. Unión Temporal Rastrojo a Escala Humana HINSAT. Secretaría de Ambiente. 130 pp.

Primack, R. F. 2001. Problemas de las poblaciones pequeñas. Capítulo 11. En: Primack, R., R. Rozzi, P. Feinsinger, R. Dirzo y F. Massardo (Eds). Fundamentos de conservación biológica. Perspectivas latinoamericanas. Fondo de cultura económica. México D.F.

Remolina, F. 2007. Procesos de fragmentación en los Cerros Orientales de Bogotá. Informe final contrato de prestación de servicios No. 144 de 2006. Jardín Botánico de Bogotá.

Remolina, F. 2011. Propuesta de estructura ecológica regional de la región capital y guía técnica para su declaración y consolidación. Contrato interadministrativo de ciencia y tecnología no. 1392 de 2009 entre la Universidad Distrital Francisco José De Caldas y la Secretaría Distrital De Ambiente. 121 pp.

Sguerra, S., P. Bejarano, O. Rodríguez, J. Blanco, O. Jaramillo y G. Sanclemente. 2011. Corredor de conservación Chingaza - Sumapaz - Querrero.
Resultados del diseño y lineamientos de acción. Conservación Internacional Colombia y Empresa de Acueducto y Alcantarillado de Bogotá ESP. Bogotá, Colombia. 184 pp.

Sieving, K. E., M. F. Willson y T. L. De Santo. 2000. Defining corridors functions for endemic birds in fragmented south-temperate rainforest. Conservation Biology 14: 1120-1132.

Tambosi, L. R. y J. P. Metzger. 2013. A framework for setting local restoration priorities based on landscape context. Natureza \& Consevação 11: 152-157.

Tambosi L. R., A. C. Martensen, M. C. Ribeiro y J. P. Metzger. 2014. A framework to optimize biodiversity restoration efforts based on habitat amount and landscape connectivity. Restoration Ecology 22: 169-177.

Taylor, P. D., L. Fahrig, K. Henein y G. Merriam. 1993. Connectivity is a vital element of landscape structure. Oikos 68: 571-573.

Taylor, P., L. Fahrig y K. With. 2006. Landscape connectivity: A return to basics. Pp. 29-43 En: Crooks K. R. y M. Sanjayan (Eds.). Connectivity Conservation. Maintaining connections for nature. Cambridge University Press, Cambridge, UK.

Tracey, J. A. 2006. Individual-based modeling as a tool for conserving connectivity. Pp. 343-368. En: Crooks, K. R. y M. Sanjayan (Eds.). Connectivity Conservation. Maintaining connections for nature. Cambridge University Press, Cambridge, UK.

Theobald, D. M. 2006. Exploring the functional connectivity of landscapes using landscape networks. Pp. 416-443. En: Crooks K. R. y M. Sanjayan (Eds.). Connectivity conservation: Maintaining connections for nature. Cambridge University Press, Cambridge, UK.

Trujillo, L. 2012. Caracterización florística y estructural e identificación, distribución y espacialización de comunidades vegetales en las áreas priorizadas por el Jardín Botánico para la conservación en Bogotá D.C. y la región. Contrato 811. Jardín Botánico de Bogotá José Celestino Mutis.

Urban, D. y T. Keitt. 2001. Landscape connectivity: a graph-theoretic perspective. Ecology 82: 1205-1218.

Vargas, W. y W. Ramírez. 2014. Lineamientos generales para la restauración del bosque seco tropical en Colombia. Capítulo 9. En: Pizano, C. y H. García (Eds.). El Bosque Seco Tropical en Colombia. Instituto de Investigación de recursos biológicos Alexander von Humboldt. Bogotá. D.C. 
Paola Johanna Isaacs-Cubides

Instituto de Investigación de Recursos Biológicos Alexander von Humboldt,

Programa Gestión Territorial de la Biodiversidad, Bogotá, Colombia

pisaacs@humboldt.org.co

Ledy N. Trujillo-Ortiz

Investigadora independiente,

Bogotá, Colombia

ledytrujillo@yahoo.es

\section{Vilma Jaimes}

Investigadora independiente,

Bogotá, Colombia

vjaimes.s@gmail.com
Zonificación de alternativas de conectividad ecológica, restauración y conservación en las microcuencas Curubital, Mugroso, Chisacá y Regadera, cuenca del río Tunjuelo (Distrito Capital de Bogotá), Colombia

Citación del artículo: Isaac-Cubides, P. J., L. N. Trujillo y V. Jaimes. 2017. Zonificación de alternativas de conectividad ecológica, restauración y conservación en las microcuencas Curubital, Mugroso, Chisacá y Regadera, cuenca del río Tunjuelo (Distrito Capital de Bogotá), Colombia. Biota Colombiana 18 (Suplemento 1): 70-88. DOI: 10.21068/ c2017.v18s01a04

Recibido: 26 de agosto de 2014

Aprobado: 10 de enero de 2017 


\title{
Guía para autores
}

\author{
(humboldt.org.co/es/bibliotecaypublicaciones/biota)
}

\section{Preparación del manuscrito}

El envío de un manuscrito implica la declaración explícita por parte del autor(es) de que este no ha sido previamente publicado, ni aceptado para su publicación en otra revista $u$ otro órgano de difusión científica. Todas las contribuciones son de la entera responsabilidad de sus autores y no del Instituto de Investigación de Recursos Biológicos Alexander von Humboldt, ni de la revista o sus editores.

Los trabajos pueden estar escritos en español, inglés o portugués, y se recomienda que no excedan las 40 páginas (párrafo espaciado a 1,5 líneas) incluyendo tablas, figuras y anexos. En casos especiales el editor podrá considerar la publicación de trabajos más extensos, monografías o actas de congresos, talleres o simposios. De particular interés para la revista son las descripciones de especies nuevas para la ciencia, nuevos registros geográficos y listados de la biodiversidad regional.

Para la elaboración de los textos del manuscrito se puede usar cualquier procesador de palabras (preferiblemente Word); los listados (a manera de tabla) deben ser elaborados en una hoja de cálculo (preferiblemente Excel). Para someter un manuscrito es necesario además anexar una carta de intención en la que se indique claramente:

1. Nombre completo del (los) autor (es), y direcciones para envío de correspondencia (es indispensable suministrar una dirección de correo electrónico para comunicación directa).

2. Título completo del manuscrito.

3. Nombres, tamaños y tipos de archivos suministrados.

4. Lista mínimo de tres revisores sugeridos que puedan evaluar el manuscrito, con sus respectivas direcciones electrónicas.

\section{Evaluación del manuscrito}

Los manuscritos sometidos serán revisados por pares científicos calificados, cuya respuesta final de evaluación puede ser: a) aceptado (en cuyo caso se asume que no existe ningún cambio, omisión o adición al artículo, y que se recomienda su publicación en la forma actualmente presentada); b) aceptación condicional (se acepta y recomienda el artículo para su publicación solo si se realizan los cambios indicados por el evaluador); y c) rechazo (cuando el evaluador considera que los contenidos o forma de presentación del artículo no se ajustan a los requerimientos y estándares de calidad de Biota Colombiana).

\section{Texto}

- Para la presentación del manuscrito configure las páginas de la siguiente manera: hoja tamaño carta, márgenes de $2,5 \mathrm{~cm}$ en todos los lados, interlineado 1,5 y alineación hacia la izquierda (incluyendo título y bibliografía).

- Todas las páginas de texto (a excepción de la primera correspondiente al título), deben numerarse en la parte inferior derecha de la hoja.
- Use letra Times New Roman o Arial, tamaño 12 puntos en todos los textos. Máximo 40 páginas, incluyendo tablas, figuras y anexos. Para tablas cambie el tamaño de la fuente a 10 puntos. Evite el uso de negritas o subrayados.

- Los manuscritos debe llevar el siguiente orden: título, resumen y palabras clave, abstract y key words, introducción, material y métodos, resultados, discusión, conclusiones (optativo), agradecimientos (optativo) y bibliografía. Seguidamente, presente una página con la lista de tablas, figuras y anexos. Finalmente, incluya las tablas, figuras y anexos en archivos separadas, debidamente identificadas.

- Escriba los nombres científicos de géneros, especies y subespecies en cursiva (itálica). Proceda de la misma forma con los términos en latín (p. e. sensu, et al.). No subraye ninguna otra palabra o título. No utilice notas al pie de página.

- En cuanto a las abreviaturas y sistema métrico decimal, utilice las normas del Sistema Internacional de Unidades (SI) recordando que siempre se debe dejar un espacio libre entre el valor numérico y la unidad de medida (p. e. $16 \mathrm{~km}, 23^{\circ} \mathrm{C}$ ). Para medidas relativas como m/seg., use m.seg-1.

- Escriba los números del uno al diez siempre con letras, excepto cuando preceden a una unidad de medida (p. e. $9 \mathrm{~cm}$ ) o si se utilizan como marcadores (p. e. parcela 2, muestra 7).

- No utilice punto para separar los millares, millones, etc. Utilice la coma para separar en la cifra la parte entera de la decimal ( $\mathrm{p}$. e. 3,1416). Enumere las horas del día de 0:00 a 24:00.

- Exprese los años con todas las cifras sin demarcadores de miles (p. e. 1996-1998). En español los nombres de los meses y días (enero, julio, sábado, lunes) siempre se escriben con la primera letra minúscula, no así en inglés.

- Los puntos cardinales (norte, sur, este y oeste) siempre deben ser escritos en minúscula, a excepción de sus abreviaturas $\mathrm{N}, \mathrm{S}$, $\mathrm{E}, \mathrm{O}$ (en inglés $\mathrm{W}$ ), etc. La indicación correcta de coordenadas geográficas es como sigue: $02^{\circ} 37^{\prime} 53^{\prime \prime} \mathrm{N}-56^{\circ} 28^{\prime} 53^{\prime \prime} \mathrm{O}$. La altitud geográfica se citará como se expresa a continuación: $1180 \mathrm{~m}$ s.n.m. (en inglés $1180 \mathrm{~m}$ a.s.l).

- Las abreviaturas se explican únicamente la primera vez que son usadas.

- Al citar las referencias en el texto mencione los apellidos de los autores en caso de que sean uno o dos, y el apellido del primero seguido por et al. cuando sean tres o más. Si menciona varias referencias, éstas deben ser ordenadas cronológicamente y separadas por comas (p. e. Rojas 1978, Bailey et al. 1983, Sephton 2001, 2001).

- Resumen: incluya un resumen de máximo 200 palabras, tanto en español o portugués como inglés.

- Palabras Clave: máximo seis palabras clave, preferiblemente complementarias al título del artículo, en español e inglés. 


\section{Agradecimientos}

Opcional. Párrafo sencillo y conciso entre el texto y la bibliografía. Evite títulos como Dr., Lic., TSU, etc.

\section{Fotografías, figuras, tablas y anexos}

Refiera las figuras (gráficas, diagramas, ilustraciones y fotografías) sin abreviación (p. e. Figura 3 ) al igual que las tablas (p. e. Tabla 1). Gráficos (p. e. CPUE anuales) y figuras (histogramas de tallas), preferiblemente en blanco y negro, con tipo y tamaño de letra uniforme. Deben ser nítidas y de buena calidad, evitando complejidades innecesarias (por ejemplo, tridimensionalidad en gráficos de barras); cuando sea posible use solo colores sólidos en lugar de tramas. Las letras, números o símbolos de las figuras deben ser de un tamaño adecuado de manera que sean claramente legibles una vez reducidas. Para el caso de las fotografías y figuras digitales es necesario que estas sean guardadas como formato tiff con una resolución de 300 dpi. Es oportuno que indique en qué parte del texto desea insertarla.

Lo mismo aplica para las tablas y anexos, los cuales deben ser simples en su estructura (marcos) y estar unificados. Presente las tablas en archivo aparte (Excel), identificadas con su respectivo número. Haga las llamadas a pie de página de tabla con letras ubicadas como superíndice. Evite tablas grandes sobrecargadas de información y líneas divisorias o presentadas en forma compleja. Es oportuno que indique en qué parte del texto desea insertar tablas y anexos.

\section{Bibliografía}

Contiene únicamente la lista de las referencias citadas en el texto. Ordénelas alfabéticamente por autores y cronológicamente para un mismo autor. Si hay varias referencias de un mismo autor(es) en el mismo año, añada las letras a, b, c, etc. No abrevie los nombres de las revistas. Presente las referencias en el formato anexo, incluyendo el uso de espacios, comas, puntos, mayúsculas, etc.

\section{ARTíCUlO EN REVISTAS}

Agosti, D., C. R. Brandao y S. Diniz. 1999. The new world species of the subfamily Leptanilloidinae (Hymenoptera: Formicidae). Systematic Entomology 24: 14-20.

LIBROS, TESIS E INFORMES TÉCNICOS

Libros: Gutiérrez, F. P. 2010. Los recursos hidrobiológicos y pesqueros en Colombia. Instituto de Investigación de Recursos Biológicos Alexander von Humboldt. Bogotá, D. C., 118 pp.

Tesis: Cipamocha, C. A. 2002. Caracterización de especies y evaluación trófica de la subienda de peces en el raudal Chorro de Córdoba, bajo río Caquetá, Amazonas, Colombia. Trabajo de grado. Universidad Nacional de Colombia, Facultad de Ciencias, Departamento de Biología. Bogotá D. C., 160 pp.

Informes técnicos: Andrade, G. I. 2010. Gestión del conocimiento para la gestión de la biodiversidad: bases conceptuales y propuesta programática para la reingeniería del Instituto Humboldt. Informe Técnico. Instituto de Investigación de Recursos Biológicos Alexander von Humboldt. Bogotá D. C., 80 pp.

Capitulo en libro o en informe: Fernández F., E. E. Palacio y W. P. MacKay. 1996. Introducción al estudio de las hormigas (Hymenoptera: Formicidae) de Colombia. Pp: 349-412. En: Amat, G. D., G. Andrade y F. Fernández (Eds.). Insectos de Colombia. Estudios Escogidos. Academia Colombiana de Ciencias Exactas, Físicas y Naturales \& Centro Editorial Javeriano, Bogotá.

Resumen en congreso, simposio, talleres: Señaris, J. C. 2001. Distribución geográfica y utilización del hábitat de las ranas de cristal (Anura; Centrolenidae) en Venezuela. En: Programa y Libro de Resúmenes del IV Congreso Venezolano de Ecología. Mérida, Venezuela, p. 124.

PÁginas Web

No serán incluidas en la bibliografía, sino que se señalarán claramente en el texto al momento de mencionarlas.

\section{Guidelines for authors}

(humboldt.org.co/es/bibliotecaypublicaciones/biota)

\section{Manuscript preparation}

Submitting a manuscript implies the explicit statement by the author(s) that the paper has not been published before nor accepted for publication in another journal or other means of scientific diffusion. Contributions are entire responsibility of the author and not the Alexander von Humboldt Institute for Research on Biological Resources, or the journal and their editors.

Papers can be written in Spanish, English or Portuguese and it is recommended not exceeding 40 pages (with paragraphs spaced at 1,5) including tables, figures and Annex. For special cases, the editor could consider publishing more extensive papers, monographs or symposium conclusions. New species descriptions for science, new geographic records and regional biodiversity lists are of particular interest for this journal.
Any word-processor program may be used for the text (Word is recommended). taxonomic list or any other type of table, should be prepared in spreadsheet aplication (Excel is recommended). To submit a manuscript must be accompanied by a cover letter which clearly indicate s:

1. Full names, mailing addresses and e-mail addresses of all authors. (Please note that email addresses are essential to direct communication).

2. The complete title of the article.

3. Names, sizes, and types of files provide.

4. A list of the names and addresses of at least three (3) reviewers who are qualified to evaluate the manuscript. 


\section{Evaluation}

Submitted manuscript will have a peer review evaluation. Resulting in any of the following: a) accepted (in this case we assume that no change, omission or addition to the article is required and it will be published as presented.); b) conditional acceptance (the article is accepted and recommended to be published but it needs to be corrected as indicated by the reviewer); and c) rejected (when the reviewer considers that the contents and/or form of the paper are not in accordance with requirements of publication standards of Biota Colombiana).

\section{Text}

- The manuscript specifications should be the following: standard letter size paper, with $2.5 \mathrm{~cm}$ margins on all sides, 1.5-spaced and left-aligned (including title and bibliography)

- All text pages (with the exception of the title page) should be numbered. Pages should be numbered in the lower right corner.

- Use Times New Roman or Arial font, size 12, for all texts. Use size 10 text in tables. Avoid the use of bold or underlining. 40 pages maximum, including tables, figures and annex. For tables use size 10 Times New Roman or Arial Font (the one used earlier).

- The manuscripts must be completed with the following order: title, abstract and key words, then in Spanish Título, Resumen y Palabras claves. Introduction, Materials and Methods, Results, Discussion, conclusions (optional), acknowledgements (optional) and bibliography. Following include a page with the Table, Figure and Annex list. Finally tables, figures and annex should be presented and clearly identified in separate tables.

- Scientific names of genera, species and subspecies should be written in italic. The same goes for Latin technical terms (i.e sensu, et al.). Avoid the use of underlining any word or title. Do not use footnotes.

- As for abbreviations and the metric system, use the standards of the International System of Units (SI) remembering that there should always be a space between the numeric value and the measure unit (e.g., $16 \mathrm{~km}, 23^{\circ} \mathrm{C}$ ). For relative measures such as $\mathrm{m} / \mathrm{sec}$, use $\mathrm{m} \cdot \mathrm{sec}^{-1}$.

- Write out numbers between one to ten in letters except when it precedes a measure unit (e.g., $9 \mathrm{~cm}$ ) or if it is used as a marker (e.g., lot 9, sample 7).

- Do not use a point to seperate thousands, millions, etc. Use a comma to separate the whole part of the decimal (e.g., 3,1416). Numerate the hours of the from 0:00 to 24:00. Express years with all numbers and without marking thousands (e.g., 19961998). In Spanish, the names of the months and days (enero, julio, sábado, lunes) are always written with the first letter as a lower case, but it is not this way in English.

- The cardinal points (north, south, east, and west) should always be written in lower case, with the excpetino of abbreviations $\mathrm{N}, \mathrm{S}, \mathrm{E}, \mathrm{O}$ (in English NW), etc. The correct indication of geographic coordinates is as follows: $02^{\circ} 37^{\prime} 53^{\prime \prime} \mathrm{N}-56^{\circ} 28^{\prime} 53^{\prime \prime} \mathrm{O}$. The geographic altitude should be cited as follows: $1180 \mathrm{~m}$ a.s.l.

- Abbreviations are explained only the first time they are used.
- When quoting references in the text mentioned author's last names when they are one or two, and et al. after the last name of the first author when there are three or more. If you mention many references, they should be in chronological order and separated by commas (e.g., Rojas 1978, Bailey et al. 1983, Sephton 2001, 2001).

- Abstract: include an abstract of 200 words maximum, in Spanish, Portuguese or English.

- KeY WORDS: six key words maximum, complementary to the title.

\section{Pictures, Figures, Tables and Annex}

- Figures (graphics, diagrams, illustrations and photographs) without abbreviation (e.g. Figure 3) the same as tables (e.g., Table 1). Graphics and figures should be in black and white, with uniform font type and size. They should be sharp and of good quality, avoiding unnecessary complexities (e.g., three dimensions graphics). When possible use solid color instead of other schemes. The words, numbers or symbols of figures should be of an adequate size so they are readable once reduced. Digital figures must be sent at $300 \mathrm{dpi}$ and in .tiff format. Please indicate in which part of the text you would like to include it.

- The same applies to tables and annexes, which should be simple in structure (frames) and be unified. Present tables in a separate file (Excel), identified with their respective number. Make calls to table footnotes with superscript letters above. Avoid large tables of information overload and fault lines or presented in a complex way. It is appropriate to indicate where in the text to insert tables and annexes.

\section{Bibliography}

References in bibliography contains only the list of references cited in the text. Sort them alphabetically by authors and chronologically by the same author. If there are several references by the same author(s) in the same year, add letters a, b, c, etc. Do not abbreviate journal names. Present references in the attached format, including the use of spaces, commas, periodss, capital letters, etc.

JOURNAL ARTICLE

Agosti, D., C. R. Brandao y S. Diniz. 1999. The new world species of the subfamily Leptanilloidinae (Hymenoptera: Formicidae). Systematic Entomology 24: 14-20.

BOOK, THESIS, TECHNICAL REVIEWS

Book: Gutiérrez, F. P. 2010. Los recursos hidrobiológicos y pesqueros en Colombia. Instituto de Investigación de Recursos Biológicos Alexander von Humboldt. Bogotá, D. C. 118 pp.

Thesis: Cipamocha, C. A. 2002. Caracterización de especies y evaluación trófica de la subienda de peces en el raudal Chorro de Córdoba, bajo río Caquetá, Amazonas, Colombia. Trabajo de grado. Universidad Nacional de Colombia, Facultad de Ciencias, Departamento de Biología. Bogotá D. C. 160 pp.

Technical reviews: Andrade, G. I. 2010. Gestión del conocimiento para la gestión de la biodiversidad: bases conceptuales y propuesta programática para la reingeniería del Instituto Humboldt. Informe 
Técnico. Instituto de Investigación de Recursos Biológicos Alexander von Humboldt. Bogotá D. C. 80 pp.

Book chapter or in review: Fernández F., E. E. Palacio y W. P. MacKay. 1996. Introducción al estudio de las hormigas (Hymenoptera: Formicidae) de Colombia. Pp:349-412. En: Amat, G. D., G. Andrade y F. Fernández (Eds.). Insectos de Colombia. Estudios Escogidos. Academia Colombiana de Ciencias Exactas, Físicas y Naturales \& Centro Editorial Javeriano, Bogotá.
Symposium abstract: Señaris, J. C. 2001. Distribución geográfica y utilización del hábitat de las ranas de cristal (Anura; Centrolenidae) en Venezuela. En: Programa y Libro de Resúmenes del IV Congreso Venezolano de Ecología. Mérida, Venezuela, p. 124.

WeB PAGES

Not be included in the literature, but clearly identified in the text at the time of mention.

\section{Guía para autores - Artículos de Datos}

www.humboldt.org.co/es/bibliotecaypublicaciones/biota- biotacol@humboldt.org.co

www.sibcolombia.net - sib+iac@humboldt.org.co

El objetivo de esta guía es establecer y explicar los pasos necesarios para la elaboración de un manuscrito con el potencial de convertirse en artículo de datos para ser publicado en la revista Biota Colombiana. En esta guía se incluyen aspectos relacionados con la preparación de datos y el manuscrito.

\section{¿Qué es un artículo de datos?}

Un artículo de datos o Data Paper es un tipo de publicación académica que ha surgido como mecanismo para incentivar la publicación de datos sobre biodiversidad, a la vez que es un medio para generar reconocimiento académico y profesional adecuado a todas las personas que intervienen de una manera u otra en la gestión de información sobre biodiversidad.

Los artículos de datos contienen las secciones básicas de un artículo científico tradicional. Sin embargo, estas se estructuran de acuerdo a un estándar internacional para metadatos (información que le da contexto a los datos) conocido como el GBIF Metadata Profile (GMP) ${ }^{1}$. La estructuración del manuscrito con base en este estándar se da, en primer lugar, para facilitar que la comunidad de autores que publican conjuntos de datos a nivel global, con presencia en redes como la Global Biodiversity Information Facility (GBIF) y otras redes relacionadas, puedan publicar fácilmente artículos de datos obteniendo el reconocimiento adecuado a su labor. En segundo lugar, para estimular que los autores de este tipo de conjuntos de datos que aún no han publicado en estas redes de información global, tengan los estímulos necesarios para hacerlo.

Un artículo de datos debe describir de la mejor manera posible el quién, qué, dónde, cuándo, por qué y cómo de la toma y almacenamiento de los datos, sin llegar a convertirse en el medio para realizar un análisis exhaustivo de los mismos, como sucede en otro tipo de publicaciones académicas. Para profundizar en este modelo de publicación se recomienda consultar a Chavan y Penev $(2011)^{2}$.

\begin{abstract}
¿Qué manuscritos pueden llegar a ser artículos de datos?
Manuscritos que describan conjuntos de datos primarios y originales que contengan registros biológicos (captura de datos de la presencia de un(os) organismo(s) en un lugar y tiempo determinados); información asociada a ejemplares de colecciones biológicas; listados temáticos o geográficos de especies; datos genómicos y todos aquellos datos que sean susceptibles de ser estructurados con el estándar Darwin Core ${ }^{3}$ (DwC). Este estándar es utilizado dentro de la comunidad de autores que publican conjuntos de datos sobre biodiversidad para estructurar los datos y de esta manera poder consolidarlos e integrarlos desde diferentes fuentes a nivel global. No se recomienda someter manuscritos que describan conjuntos de datos secundarios, como por ejemplo compilaciones de registros biológicos desde fuentes secundarias (p.e. literatura o compilaciones de registros ya publicados en redes como GBIF o IABIN).
\end{abstract}

\section{Preparación de los datos}

Como se mencionó anteriormente los datos sometidos dentro de este proceso deben ser estructurados en el estándar DwC. Para facilitar su estructuración, el Sistema de Información sobre Biodiversidad de Colombia (SiB Colombia), ha creado dos plantillas en Excel, una para registros biológicos y otra para listas de especies. Lea y siga detenidamente las instrucciones de las plantillas para la estructuración de los datos a publicar. Para cualquier duda sobre el proceso de estructuración de estos datos por favor contactar al equipo coordinador del SiB Colombia (ECSiB) en sib+iac@humboldt.org.co.

\footnotetext{
${ }^{1}$ Wieczorek, J. 2011. Perfil de Metadatos de GBIF: una guía de referencia rápida. En: Wieczorek, J. The GBIF Integrated Publishing Toolkit User Manual, version 2.0. Traducido y adaptado del inglés por D. Escobar. Sistema de Información sobre Biodiversidad de Colombia, Bogotá D.C., Colombia, 23p. Disponible en http://www.sibcolombia.net/repositorio-de-documentos.

${ }^{2}$ Chavan, V. y L. Penev. 2011. The data paper: The mechanism to incentivize data publishing in biodiversity science. BMC Bioinformatics 12 (Suppl 15): $\mathrm{S} 2$.

${ }^{3}$ TDWG. 2011. Darwin Core: una guía de referencia rápida. (Versión original producida por TDWG, traducida al idioma español por Escobar, D.; versión 2.0). Bogotá: SiB Colombia, 33 pp. Disponible en http://www.sibcolombia.net/repositorio-de-documentos
} 


\section{Preparación del manuscrito}

Para facilitar la creación y estructuración del manuscrito en el estándar GMP, se cuenta con la ayuda de un editor electrónico (http://ipt.sibcolombia.net/biota) que guiará al autor en dicho proceso y que finalmente generará una primera versión del manuscrito. Se recomienda el uso del manual GMP, como una guía de la información a incluir en cada sección del manuscrito, junto con el anexo 1.

Pasos a seguir para la elaboración del manuscrito:

1 Solicitealcorreosib+iac@humboldt.org.coelaccesoaleditor electrónico. El EC-SiB le asignará un usuario y contraseña.

2. Ingreseconsuusuarioycontraseñaaleditorelectrónico,luego diríjase a la pestaña Gestión de recursos y cree un nuevo recurso asignando un nombre corto a su manuscrito usando el formato "AcrónimoDeLaInstitución_año_tipoDeConjuntoDeDatos", p.e. ABC_2010_avestinije y dar clic en el botón crear.

3. Enlavistageneraldeleditorseleccione "editar"enlapestaña Metadatos (por favor, no manipule ningún otro elemento), allí encontrará diferentes secciones (panel derecho) que lo guiarán en la creación de su manuscrito. Guarde los cambios al finalizar cada sección, de lo contrario perderá la información. Recuerde usar el manual GMP. A continuación se presentan algunas recomendaciones para la construcción del manuscrito. Las secciones se indican en MAYUSCULAS y los elementos de dichas secciones en negrilla.

- $\quad$ En PARTES ASOCIADAS incluya únicamente aquellas personas que no haya incluido en INFORMACIÓN BÁSICA.

- Los DATOS DEL PROYECTO y DATOS DE LA COLECCIÓN son opcionales según el tipo de datos. En caso de usar dichas secciones amplíe o complemente información ya suministrada, p. ej. no repita información de la descripción (COBERTURA GEOGRÁFICA) en la descripción del área de estudio (DATOS DEL PROYECTO).

- De igual manera, en los MÉTODOS DE MUESTREO, debe ampliar o complementar información, no repetirla. La información del área de estudio debe dar un contexto específico a la metodología de muestreo.

- Es indispensable documentar el control de calidad en MÉTODOS DE MUESTREO. Acá se debe describir que herramientas o protocolos se utilizaron para garantizar la calidad y coherencia de los datos estructurados con el estándar DwC.

- Para crear la referencia del recurso, en la sección REFERENCIAS, utilice uno de los dos formatos propuestos (Anexo 2). No llene el identificador de la referencia, este será suministrado posteriormente por el EC-SiB.

- Para incluir la bibliografía del manuscrito en referencias, ingrese cada una de las citas de manera individual, añadiendo una nueva referencia cada vez haciendo clic en la esquina inferior izquierda.

4. Rectifique que el formato de la información suministrada cumpla con los lineamientos de la revista (p. ej. abreviaturas, unidades, formato de números etc.) en la Guía general para autores de Biota Colombiana.

5. Una vez incluida y verificada toda la información en el editor electrónico notifique al EC-SiB al correo electrónico sib+iac@ humboldt.org.co, indicando que ha finalizado la edición del manuscrito. Adicionalmente adjunte la plantilla de Excel con los datos estructurados (elimine todas las columnas que no utilizó). El EC-SiB realizará correcciones y recomendaciones finales acerca de la estructuración de los datos y dará las instrucciones finales para que usted proceda a someter el artículo.

\section{Someter el manuscrito}

Una vez haya terminado la edición de su manuscrito y recibido las instrucciones por parte del EC-SIB, envíe una carta al correo electrónico biotacol@humboldt.org.co para someter su artículo, siguiendo las instrucciones en la Guía general para autores de Biota Colombiana.

Recuerde adjuntar:

- Plantilla de Excel con la última versión de los datos revisada por el EC-SiB.

- Documento de Word con las figuras y tablas seguidas de una lista las mismas.

Cuando finalice el proceso, sus datos se harán públicos y de libre acceso en los portales de datos del SiB Colombia y GBIF. Esto permitirá que sus datos estén disponibles para una audiencia nacional e internacional, manteniendo siempre el crédito para los autores e instituciones asociadas. 
Anexo 1. Estructura base de un artículo de datos y su correspondencia con el editor electrónico basado en el GMP.

\begin{tabular}{|c|c|}
\hline SECCIÓN/SUBSECCIÓN & CORRESPONDENCIA CON LOS ELEMENTOS DEL EDITOR ELECTRÓNICO \\
\hline TÍTUlO & Derivado del elemento título. \\
\hline AutORES & Derivado de los elementos creador del recurso, proveedor de los metadatos y partes asociadas. \\
\hline AfILIACIONES & $\begin{array}{l}\text { Derivado de los elementos creador del recurso, proveedor de los metadatos y partes asociadas. } \\
\text { De estos elementos, la combinación de organización, dirección, código postal, ciudad, país y } \\
\text { correo electrónico, constituyen la afiliación. }\end{array}$ \\
\hline AUTOR DE CONTACTO & Derivado de los elementos creador del recurso y proveedor de los metadatos. \\
\hline CitACiÓN & Para uso de los editores. \\
\hline CitACión DELE RECURSO & Derivada del elemento referencia del recurso. \\
\hline RESUMEN & Derivado del elemento resumen. Máximo 200 palabras. \\
\hline Palabras Clave & Derivadas del elemento palabras clave. Máximo seis palabras. \\
\hline ABSTRACT & Derivado del elemento abstract. Máximo 200 palabras. \\
\hline KEY WORDS & Derivadas del elemento key words. Máximo seis palabras. \\
\hline INTRODUCCIÓN & $\begin{array}{l}\text { Derivado del elemento propósito (de las secciones Introducción y Antecedentes). Se sugiere un } \\
\text { breve texto para introducir las siguientes secciones. Por ejemplo, historia o contexto de la colección } \\
\text { biológica o proyecto en relación con los datos descritos, siempre y cuando no se repita información } \\
\text { en las subsecuentes secciones. }\end{array}$ \\
\hline Datos del proyecto & $\begin{array}{l}\text { Derivada de los elementos de la sección Datos del proyecto: título, nombre, apellido, rol, fuentes } \\
\text { de financiación, descripción del área de estudio y descripción del proyecto. }\end{array}$ \\
\hline Cobertura taxonómica & $\begin{array}{l}\text { Derivada de los elementos de la sección Cobertura taxonómica: descripción, nombre científico, } \\
\text { nombre común y categoría. }\end{array}$ \\
\hline Cobertura geográfica & $\begin{array}{l}\text { Derivada de los elementos de la sección Cobertura geográfica: descripción, latitud mínima, } \\
\text { latitud máxima, longitud mínima, longitud máxima. }\end{array}$ \\
\hline Cobertura temporal & Derivada de los elementos de la sección Cobertura temporal: tipo de cobertura temporal. \\
\hline Datos de la colección & $\begin{array}{l}\text { Derivada de los elementos de la sección Datos de la colección: nombre de la colección, } \\
\text { identificador de la colección, identificador de la colección parental, método de preservación } \\
\text { de los especímenes y unidades curatoriales. }\end{array}$ \\
\hline MATERIAL Y MÉTODOS & $\begin{array}{l}\text { Derivado de los elementos de la sección Métodos de muestreo: área de estudio, descripción del } \\
\text { muestreo, control de calidad, descripción de la metodología paso a paso. }\end{array}$ \\
\hline \multicolumn{2}{|r|}{ ( } \\
\hline Descripción del conjunto de datos & $\begin{array}{l}\text { Derivado de los elementos de las secciones Discusión y Agradecimientos, contiene información } \\
\text { del formato de los datos y metadatos: nivel de jerarquía, fecha de publicación y derechos de } \\
\text { propiedad intelectual. }\end{array}$ \\
\hline Discusión & $\begin{array}{l}\text { Se deriva del elemento discusión. Un texto breve (máximo } 500 \text { palabras), que puede hacer } \\
\text { referencia a la importancia, relevancia, utilidad o uso que se le ha dado o dará a los datos en } \\
\text { publicaciones existentes o en posteriores proyectos. }\end{array}$ \\
\hline AgRAdECIMIENTOS & Se deriva del elemento agradecimientos. \\
\hline BIBLIOGRAFÍA & Derivado del elemento bibliografía. \\
\hline
\end{tabular}


Guía para autores - Artículos de Datos / Guidelines for authors - Data Papers

Anexo 2. Formatos para llenar el elemento referencia del recurso.

La referencia del recurso es aquella que acompañará los datos descritos por el artículo, públicos a través de las redes SiB Colombia y GBIF. Tenga en cuenta que esta referencia puede diferir de la del artículo. Para mayor información sobre este elemento contacte al EC-SiB. Aquí se sugieren dos formatos, sin embargo puede consultar otros formatos establecidos por $\mathrm{GBIF}^{4}$.

\begin{tabular}{ll}
\hline TIPO DE RECURSO & \multicolumn{1}{c}{ PLANTILLA } \\
\hline El conjunto de datos que & $<$ Institución publicadora/Grupo de investigación $>$ \\
el manuscrito describe es & $<($ Año $)>,<$ Título del recurso/Artículo $>$. $<$ Número \\
resultado de un proyecto & total de registros $>,<$ aportados por: $><$ parte \\
de carácter institucional & asociada $1($ rol $)$, parte asociada $2($ rol $)(\ldots)>$. $<$ En \\
o colectivo con múltiples & línea, $><$ url del recurso $>$. $<$ Publicado el DD/MM/ \\
participantes. & AAAA $>$
\end{tabular}

El conjunto de datos que el manuscrito describe es resultado de una iniciativa personal o de un grupo de investigación definido.
$<$ Parte asociada 1, parte asociada $2(\ldots)>$ $<$ (Año) $>,<$ Título del recurso/Artículo $>$, $<$ Número total de registros $>$, $<$ en línea, $><$ url del recurso $>$. $<$ Publicado el DD/MM/AAAA $>$

\section{EJEMPLO}

Centro Nacional de Biodiversidad (2013). Vertebrados de la cuenca de la Orinoquia. 1500 registros, aportados por Pérez, S. (Investigador principal, proveedor de contenidos, proveedor de metadatos), M. Sánchez (Procesador), D. Valencia (Custodio, proveedor de metadatos), R. Rodríguez (Procesador), S. Sarmiento (Publicador), V. B. Martínez (Publicador, editor). En línea, http://ipt.sibcolombia.net/biota/resource.do? $\mathrm{r}=$ verte_orin, publicado el 01/09/2013.

Valencia, D., R. Rodríguez y V. B. Martínez (2013). Vertebrados de la cuenca del Orinoco. 1500 registros, en línea, http://ipt.sibcolombia.net/biota/ resource.do?r=verte_orin. Publicado el 01/09/2001.

\section{Guidelines for authors - Data Papers}

www.humboldt.org.co/es/bibliotecaypublicaciones/biota-biotacol@humboldt.org.co|

www.sibcolombia.net - sib+iac@humboldt.org.co

The purpose of this guide is to establish and explain the necessary steps to prepare a manuscript with the potential to become a publishable data paper in Biota Colombiana. This guide includes aspects related to the preparation of both data and the manuscript.

\section{What is a Data Paper?}

A data paper is a scholarly publication that has emerged as a mechanism to encourage the publication of biodiversity data as well as an approach to generate appropriate academic and professional recognition to all those involved in in the management of biodiversity information.

A data paper contains the basic sections of a traditional scientific paper. However, these are structured according to an international standard for metadata (information that gives context to the data) known as the GBIF Metadata Profile (GMP) ${ }^{5}$. The structuring of the manuscript based on this standard enables the community of authors publishing datasets globally, with presence in networks such as the Global Biodiversity Information Facility (GBIF) and other related networks, to publish data easily while getting proper recognition for their work and to encourage the authors of this type of data sets that have not yet published in these global information networks to have the necessary incentives to do so.

A data paper should describe in the best possible way the Whom, What, Where, When, Why and How of documenting and recording of data, without becoming the instrument to make a detailed analysis of the data, as happens in other academic publications. To deepen this publishing model, it is recommended to consult Chavan \& Penev $(2011)^{6}$.

\footnotetext{
${ }^{4}$ GBIF (2012). Recommended practices for citation of the data published through the GBIF Network. Version 1.0 (Authored by Vishwas Chavan), Copenhagen: Global Biodiversity Information Facility. Pp.12, ISBN: 87-92020-36-4. Accessible at http://links.gbif.org/gbif_best_practice_data_citation_ en_v1

${ }^{5}$ GBIF (2011). GBIF Metadata Profile, Reference Guide, Feb 2011, (contributed by O Tuama, E., Braak, K., Copenhagen: Global Biodiversity Information Facility, 19 pp. Accesible at http://links.gbif.org/gbif_metadata_profile_how-to_en_v1.

${ }^{6}$ Chavan, V. y L. Penev. 2011. The data paper: The mechanism to incentivize data publishing in biodiversity science. BMC Bioinformatics 12 (Suppl 15): S2.
} 


\section{Which manuscripts are suitable for publication as data paper?}

Manuscripts that describe datasets containing original primary biological records (data of occurrences in a particular place and time); information associated with specimens of biological collections, thematic or regional inventories of species, genomic data and all data likely to be structured with the standard Darwin CoreDarwin Core $(\mathrm{DwC})$. This standard is used in the community of authors publishing biodiversity datasets to structure the data and thus to consolidate and integrate from different sources globally. It is not recommended to submit manuscripts describing secondary datasets, such as biological records compilations from secondary sources (e.g. literature or compilations of records already published in networks such as GBIF or IABIN).

\section{Dataset preparation}

As mentioned above data submitted in this process should be structured based on DwC standard. For ease of structuring, the Biodiversity Information System of Colombia (SiB Colombia), created two templates in Excel; one for occurrences and other for species checklist. Carefully read and follow the template instructions for structuring and publishing data. For any questions about the structure process of data please contact the Coordinator Team of SiB Colombia (EC-SiB) at sib+iac@humboldt.org.co

\section{Manuscript preparation}

To assist the creation and structuring of the manuscript in the GMP standard, an electronic writing tool is available (http://ipt. sibcolombia.net/biota) to guide the author in the process and ultimately generate a first version of the manuscript. The use of GMP manual as an information guide to include in each section of the manuscript, as well as the annex 1 is recommended.

Steps required for the manuscript preparation:

1 Request access to the electronic writing tool at sib+iac@ humboldt.org.co. The EC-SiB will assign a username and password.

2. Login to the electronic writing tool, then go to the tab Manage Resources and create a new resource by assigning a short name for your manuscript and clicking on the Create button. Use the format: "InstitutionAcronym_Year_DatasetFeature", e.g. NMNH_2010_rainforestbirds.

3. In the overview of the writing tool click on edit in Metadata section (please, do not use any other section), once there you will find different sections (right panel) that will guide you creating your manuscript. Save the changes at the end of each section, otherwise you will lose the information. Remember to use the GMP manual. Here are some recommendations for editing the metadata, sections are indicated in CAPS and the elements of these sections in bold.
- In ASSOCIATED PARTIES include only those who are not listed in BASIC INFORMATION.

- PROJECT DATA and COLLECTION DATA are optional depending on the data type. When using these sections extend or complement information already provided, i.e. do not repeat the same information describing the description (GEOGRAPHIC COVERAGE) in the study area description (PROJECT DATA).

- Likewise, in SAMPLING METHODS, you must expand or complete the information, not repeat it. The information in study extent should give a specific context of the sampling methodology.

- It is essential to document the quality control in SAMPLING METHODS. Here you should describe what tools or protocols were used to ensure the quality and consistency of data structured with DwC standard.

- To create the resource citation in the CITATIONS section, follow one of the two formats proposed (Annex 2). Do not fill out the citation identifier, this will be provided later by the EC-SiB.

- To include the manuscript bibliography in citations, enter each of the citations individually, adding a new citation each time by clicking in the bottom left.

4. Check that the format of the information provided meets the guidelines of the journal (e.g. abbreviations, units, number formatting, etc.) in the Biota Colombiana Guidelines for Authors.

5. Once included and verified all information in the writing tool, notify to EC-SiB at sib+iac@humboldt.org.co, indicating that you have finished editing the manuscript. Additionally attach the Excel template with structured data (remove all columns that were not used). The EC-SiB will perform corrections and final recommendations about the structure of the data and give you the final instructions to submit the paper.

\section{Submit the manuscript}

Once you have finished editing your manuscript and getting the instructions from EC-SIB, send a letter submitting your article to email biotacol@humboldt.org.co, following the instructions of Biota Colombiana Guidelines for Authors.

Remember to attach:

- Excel template with the latest version of the data reviewed by the EC-SiB.

- Word document with figures and tables followed by a list of them.

At the end of the process, your information will be public and freely accessible in the data portal of SiB Colombia and GBIF. This will allow your data to be available for national and international audience, while maintaining credit to the authors and partner institutions.

\footnotetext{
${ }^{7}$ Biodiversity Information Standards - TDWG. Accesible at http://rs.tdwg.org/dwc/terms/
} 
Annex 1. Basic structure of a data paper and its mapping to the writing tool elements based on GM.

\section{SECTION/SUB-SECTION HEADING \\ MAPPING WITH WRITING TOOL ELEMENTS}

TitLE Derived from the title element.

AUTHORS Derived from the resource creator, metadata provider, and associated parties elements.

AfFILIATIONS Derived from the resource creator, metadata provider and associated parties elements. From these elements combinations of organization, address, postal code, city, country and email constitute the affiliation.

CORRESPONDING AUTHOR Derived from the resource contact, metadata provider elements.

Citation For editors use.

RESOURCE CITATION Derived from the resource citation element.

RESUMEN Derived from the resumen element. 200 words max.

Palabras Clave Derived from the palabras clave element. 6 words max.

ABSTRACT Derived from the abstract element. 200 words max.

KEY WORDS Derived from the key words element. 6 words max.

INTRODUCTION Derived from the purpose (Introduction and Background section). A short text to introduce the following sections is suggested. For example, history or context of the biological collection or project related with the data described, only if that information is not present in subsequent sections.

Project data Derived from elements title, personnel first name, personnel last name, role, funding, study area description, and design description.

Taxonomic Coverage Derived from the taxonomic coverage elements: description, scientific name, common name and rank.

Geographic Coverage Derived from the geographic coverage elements: description, west, east, south, north.

Temporal Coverage Derived from the temporal coverage elements: temporal coverage type.

Collection data

MATERIALS AND METHODS

Derived from the collection data elements: collection name, collection identifier, parent collection identifier, specimen preservation method and curatorial units.

Derived from the sampling methods elements: study extent, sampling description, quality control and step description.

REsUltados

Descripción del conjunto de datos

Derived from the discussion and acknowledgments, contains information about the format of the data and metadata: hierarchy level, date published and ip rights.

Discussion Derived from the discussion element. A short text (max 500 words), which can refer to the importance, relevance, usefulness or use that has been given or will give the data in the published literature or in subsequent projects.

ACKNOWLEDGMENTS Derived from the acknowledgments element.

BiBLIOGRAPHY Derived from the citations element. 
Annex 2. Citation style quick guide for "resource reference" section.

The Resource Reference is the one that refer to the dataset described by the paper, publicly available through SiB Colombia and GBIF networks. Note that this reference may differ from the one of the paper. For more information about this element contact EC-SiB.

Here two formats are suggested; however you can consult other formats established by GBIF.

\section{TYPE OF RESOURCE}

The paper is the result of a collective or institutional project with multiple participants.

\section{TEMPLATE}

$<$ Institution/Research Group $>$. $<$ Year $>$, <Title of the Resource/Paper $>$. $<$ Number of total records $>$, <provided by :> <associated party 1 (role), associated party 2 (role), (..)>. < Online, $>$ $<$ resource URL $>$, $<$ published on $>$. $<$ Published on DD/MM/AAAA $>$.

$<$ associated party 1 , associated party $2,(\ldots)>$. $<$ Year $>$, $<$ Title of the Resource/Paper $>$, $<$ Number of total records $>,<$ Online, $><$ resource URL $>$. $<$ Published on DD/MM/AAAA $>$.

\section{EXAMPLE}

National Biodiversity (2013). Vertebrates in Orinoco, 1500 records, provided by: Perez, S. (Principal investigator, content provider), M. Sanchez (Processor), D. Valencia (Custodian Steward, metadata provider), R. Rodriguez (Processor), S. Sarmiento (Publisher), VB Martinez (Publisher, Editor). Online, http://ipt.sibcolombia.net/ biota/resource.do?r= verte_orin, published on 01/09/2013.

Valencia, D., R. Rodríguez and V. B. Martínez. (2013). Vertebrate Orinoco Basin, 1500 records, Online, http://ipt.sibcolombia.net/biota/resource. do? $\mathrm{r}=$ verte_orin, published on 01/09/2001

\footnotetext{
${ }^{8}$ GBIF (2012). Recommended practices for citation of the data published through the GBIF Network. Version 1.0 (Authored by Vishwas Chavan), Copenhagen: Global Biodiversity Information Facility. Pp.12, ISBN: 87-92020-36-4. Accessible at http://links.gbif.org/gbif_best_practice_data_citation_ en_v1
} 


\section{TABLA DE CONTENIDO / TABLE OF CONTENTS}

Presentación. Brigitte L. G. Baptiste, Carlos A. Lasso, Wilson Ramírez y Mauricio Aguilar-Garavito.

Introducción. Alianzas para la restauración de ecosistemas. I Simposio Regional de Restauración Ecológica Nodo REDCRE Suroccidente. Introduction. Alliance for restoration of ecosystems I Regional Symposium on Ecological Restoration REDCRE Node Southwest. Mauricio Aguilar-Garavito, Diana Catalina Rondón Camacho y William Vargas

Áreas naturales de bosque seco tropical en el Valle del Cauca, Colombia: una oportunidad para la restauración. Natural areas of tropical dry forest in Valle del Cauca, Colombia: an opportunity for restoration. Diana P. Alvarado-Solano y Joel T. Otero-Ospina

Lectura de un paisaje estratificado: propuesta de restauración basada en el ordenamiento multi-escala de las cañadas en la mesa de Xéridas, Santander, Colombia. Reading a stratified landscape: restoration proposal based on the multiscale ordination of the canebrakes at Mesa de Xéridas, Santander, Colombia. Germán Camargo-Ponce de León y Laura G. Agudelo-Álvarez

Experiencia piloto de nucleación con especies nativas para restaurar una zona degradada por ganadería en el norte de Antioquia, Colombia. A pilot nucleation experiment with native species to restore an area degraded by livestock in the north of Antioquia, Colombia. Mónica Díaz-Páez y Jaime Polanía .....

Zonificación de alternativas de conectividad ecológica, restauración y conservación en las microcuencas Curubital, Mugroso, Chisacá y Regadera, cuenca del río Tunjuelo (Distrito Capital de Bogotá), Colombia. Zonification of alternatives for ecological connectivity, restoration and conservation of the Curubital, Mugroso, Chisacá and Regadera microdrainages of the Tunjuelo River (Capital District of Bogotá), Colombia. Paola Isaacs Cubides, Ledy Trujillo y Vilma Jaimes

Banco de semillas de retamo espinoso Ulex europaeus L. en bordes del matorral invasor en un ecosistema zonal de bosque altoandino, Colombia. Seed bank of the spiny reed, Ulex europaeus L., along edges of thickets of this invasive species in a zonal ecosystem of High Andes forest, Colombia. Korina Ocampo-Zuleta y Jairo Solorza Bejarano .....

Forestación de bosques en sabanas de la altillanura colombiana: relevancia de las condiciones ambientales para el establecimiento de plántulas. Afforestation of savanna forests of the Colombian altillanura: relevance of the environmental conditions for the establishment of seedlings. Pablo R. Stevenson, Mónica A. Ramírez, Luisa F. Casas y Francisco Henao-Díaz 